\title{
BIOSENSORS BASED ON NANOMECHANICAL SYSTEMS
}

Javier Tamayo, Priscila M. Kosaka, José J. Ruz, Álvaro San Paulo and Montserrat Calleja*

Bionanomechanics lab, Institute of Microelectronics of Madrid, CSIC, Isaac Newton 8 (PTM), Tres Cantos, 28760 Madrid, Spain,

* Address correspondence to mcalleja@imm.cnm.csic.es

The advances in micro- and nanofabrication technologies are enabling increasingly smaller mechanical transducers capable of detecting the forces, motion, mechanical properties and masses that emerge in biomolecular interactions and fundamental biological processes. Thus, biosensors based on nanomechanical systems have gained considerable relevance in the last decade. This review provides insight into the mechanical phenomena that occur in suspended mechanical structures when either biological adsorption or interactions take place on their surface. This review guides the reader through the parameters that change as a consequence of biomolecular adsorption: mass, surface stress, effective Young's modulus and viscoelasticity. The mathematical background needed to correctly interpret the output signals from nanomechanical biosensors is also outlined here. Other practical issues reviewed are the immobilization of bioreceptor molecules on the surface of nanomechanical sensors and methods to attain that in large arrays of sensors. We describe then some relevant realizations of biosensor devices based on nanomechanical systems that harness some of the mechanical effects cited above. We finally discuss the intrinsic detection limits of the devices and the limitation that arises from non-specific adsorption. 


\section{INTRODUCTION}

\subsection{Biosensors}

A biosensor measures the physical changes that a biological recognition layer attached to a solid transducer undergoes when it interacts with a sample that contains the targeted molecules. Thus, it harnesses the capability of some biomolecules (receptors) to specifically bind (recognize) complementary biomolecules (ligands). Most typical interactions are hybridization of complementary nucleic acids and antibody/antigen binding. Biosensors are increasingly demanded in fundamental biological studies, health science research, drug discovery, and clinical diagnosis ${ }^{1-3}$. Depending on the measured physical change, biosensors can be classified into optical, electrical, and mechanical (Fig. 1a). Optical biosensors can be split into fluorescence-based detection and label-free detection. In fluorescence-based detection, either target molecules or biorecognition molecules are labeled with fluorescent tags, such as dyes; the intensity of the fluorescence signal indicates the amount of targeted molecules. While fluorescence-based detection is extremely sensitive, it suffers from laborious labeling processes that may also interfere with the function of the biomolecule. In contrast, in label-free detection, targeted molecules are not labeled or altered, and are detected in their natural forms. A significant part of label-free optical sensors measures the refractive index change near the sensor surface by exciting an evanescent field that exponentially decays into the bulk solution with a characteristic length between tens to hundreds of nanometers ${ }^{4}$. The surface plasmon resonance method is the most popular among label-free optical biosensors. Among electrical biosensors, electrochemical devices have traditionally received the major share of the attention ${ }^{5-7}$. These devices usually couple enzymes that produce or consume electrons upon substrate recognition to an electrode transducer. Many of these enzymes specifically catalyze the reactions of clinically important analytes such as glucose, lactate, cholesterol, amino acids, urate, pyruvate, glutamate, alcohol, hydroxybutyrate, to name a few. Nanotechnology advances are providing nanoscale electrical biosensors based on semiconductor nanowires and nanotubes, in which the electrochemical gating arises from a change in the local surface potential due to the target binding ${ }^{8-10}$. 
Among mechanical biosensors, quartz crystal microbalance has become one of the most established techniques ${ }^{11-13}$. These devices are based on quartz crystal resonators (such as those used in watches), that are piezoelectric and thus enable the direct measurement of the crystal deformation by electrical methods. In these devices, the resonance frequency is measured and related to the mass change induced by the analyte binding to the recognition layer immobilized on the crystal surface. A subclass of mechanical biosensors is referred to as nanomechanical biosensors, which capitalize on the nanoscale size of at least one of their dimensions ${ }^{14-20}$.

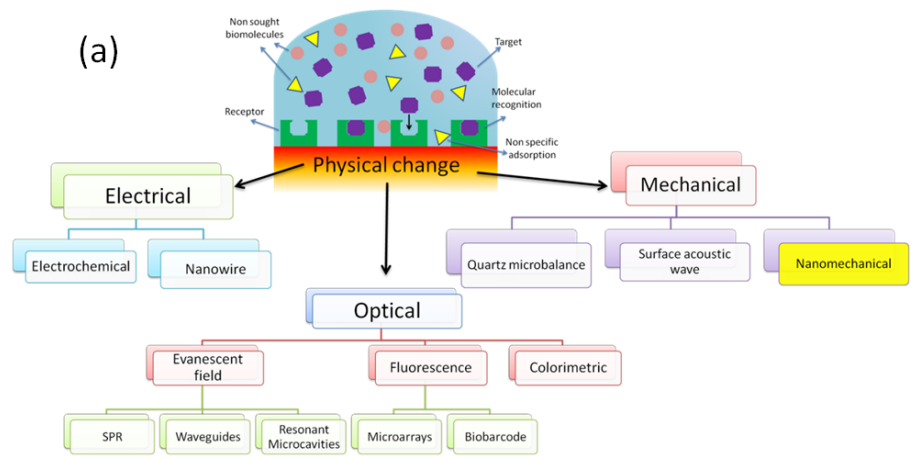

(b)

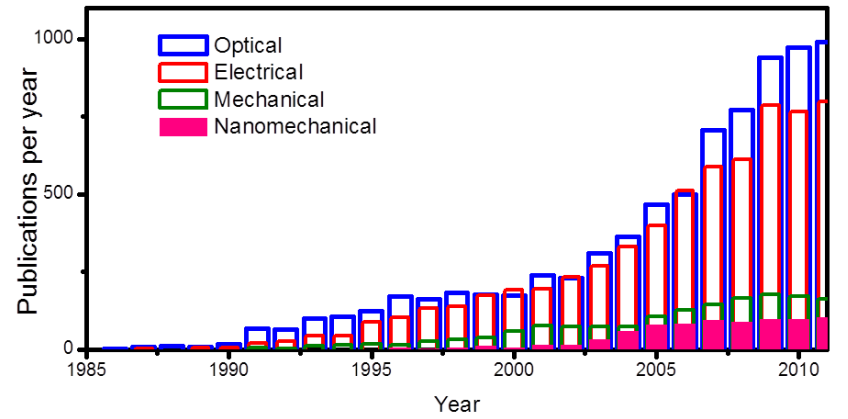

Figure 1. (a) Schematics of a biosensor and biosensor subclasses. Depending on the kind of physical change that is measured in the transducer surface, biosensors can be divided into electrical, optical and mechanical biosensors. Each category includes different type of biosensor devices. (b) Chronogram of the publication rate in optical, electrical, mechanical and nanomechanical biosensors. Up to now, optical and electrical transduction techniques clearly dominate the research on biosensors. It is expected that emerging nanomechanical biosensors will play an increasing role during the next decade.

\subsection{Why mechanics for the study of biological systems?}

Figure 1(b) shows the number of publications since 1985 related to optical, electrical mechanical and nanomechanical biosensors. The electrical and optical biosensors clearly dominate the worldwide research in biological detection. This fact is not surprising as electrical and optical transductions are versatile, mature and well-established technologies. However, there are growing evidences about the mechanical nature of many fundamental biological processes that justify the development of nanomechanical tools to sense and actuate on 
biological systems. Here we summarize a few examples of mechanotransduction processes in biology.

i) For the past fifty years, 'lock-and-key' and the 'induced fit' models has been the textbook explanation for molecular recognition events. However, recent experimental evidence supports that proteins are inherently dynamic and sample a vast ensemble of conformations, which play important roles in molecular recognition ${ }^{21-22}$ (Fig. 2(a)).

ii) Biological motors convert chemical energy to effect stepwise linear or rotary motion, and thus are essential in controlling and performing a wide variety of essential biological functions. In recent years, biomolecular motors have captivated nanotechnologist for creating sensors and actuators based on these machines ${ }^{23-26}$ (Fig. 2(b)).

iii) The shapes of the biological membranes are fashioned by the mechanical forces that operate in them ${ }^{27-28}$. Membrane curvature provides active means to control relevant functions of the cell through protein binding events. The malfunction of such proteins is implicated in disease. The energy of the protein-membrane binding is translated into mechanical membrane bending energy (Fig. 2c). Notice the beautiful analogy of this process with surface stress biosensors, in which an artificial mechanical "membrane" bends when biomolecules interact on one side of such membrane.

iv) It is increasingly evident the importance of the link between mechanics and human diseases. For instance, there is an accumulation of data in the last two decades that points out to the mechanical properties of cells as a reliable indicator of cell transformation into cancerous or metastatic cells, as well as of the effectiveness of drug treatment ${ }^{29-31}$ (Fig. 2(d)). Another relevant example concerns with viral infection. A single point mutation in the capsid protein of some viruses can significantly change the elasticity of the virus particle ${ }^{32}$. In the case of the human immunodeficiency virus, its stiffness largely decreases during the maturation process, acting as a mechanical switch in the infection process ${ }^{33}$. 


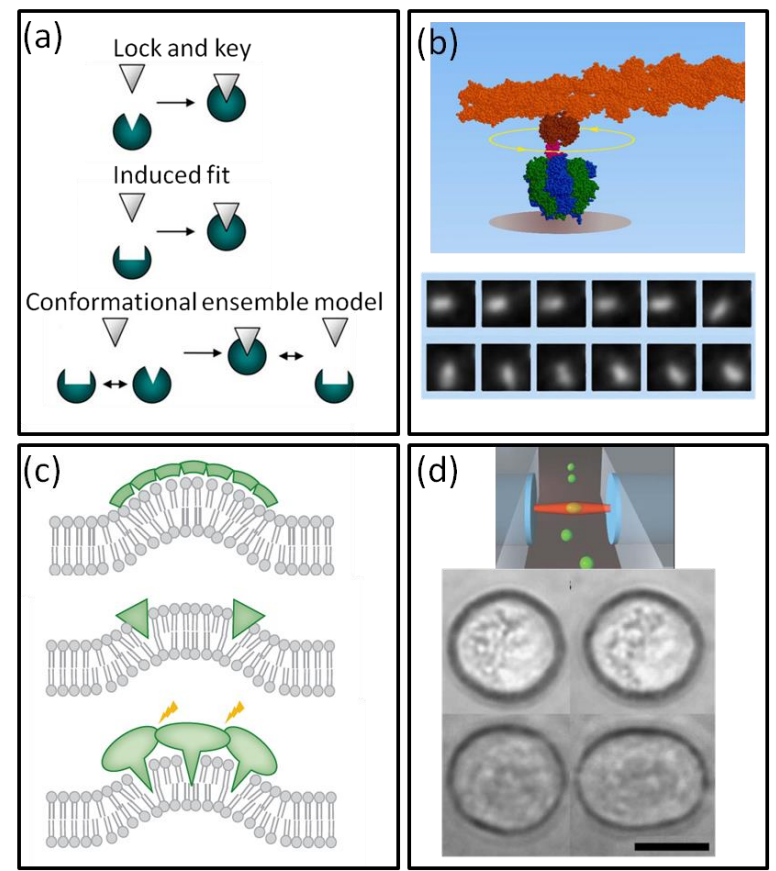

Figure 2. Examples of relevant mechanotransduction processes in biological systems. (a) Schematic of models of molecular recognition. Lock and key model: no conformational changes occur upon binding. The ligand (white) and the target (green) have complementary structures. Induced fit model: The target changes its conformation due to the interaction with the ligand. Conformational selection model: it postulates that both the ligand and the target are interconverting between an ensemble of conformations, and following binding the ensemble undergoes a population shift, redistributing the conformational states. Adapted from ${ }^{22}$ (b) The efficient conversion of chemical energy into mechanical work by specialized biological nanomachines enables a wide range of functions in nature, ranging from intracellular transport to large-scale actuation by muscles. Through suitable interfaces, these motor proteins can be employed in synthetic nanodevices with applications in biosensing, self-assembly, and molecularscale actuation. In this example, F1-ATPase, a rotary motor, which is used by nature as 'generator' to produce ATP when forced into rotation by the FO-ATPase, was labeled with a fluorescent actin, which enabled to observe its rotation, in the presence of ATP (biological fuel). The rotary torque reached more than $40 \mathrm{pN} \mathrm{nm} \mathrm{m}^{-1}$. The efficiency of this machine exceeds $50 \%$. Adapted from ${ }^{25}$ (c) Mechanism of membrane curvature upon binding of specialized proteins. Top. The rigid protein with intrinsic curvature bends the membrane. Middle. Proteins partly insert into the membrane through interaction with hydrophobic lipid groups. Bottom. Steric interactions between membranebound proteins amplify the curvature of the membrane. Adapted from ${ }^{28}$ (d). Typical examples of the stretching of breast epithelial cells. The stretching was achieved by optically induced surface forces (experimental set is sketched at the top). The images at the left column show the cells optically trapped and at the right column subject to a stretching optical force. The metastatic modMCF-7 cell (bottom) deforms more than the nonmalignant MCF-10 cell (top) under identical forces. Cancerous MCF-7 cells (not shown) exhibit an intermediate deformability. The scale bar is $10 \mu \mathrm{m}$. Adapted from ${ }^{30}$

\subsection{Nanomechanical biosensors}

Advances in micro- and nanofabrication technologies are enabling to achieve increasingly smaller mechanical transducers with micro- and nanosized moving parts whose deformation and vibration are sensitively modified upon molecular adsorption. This subcategory of mechanical biosensors is referred to as nanomechanical biosensors ${ }^{14-20,34-35}$. The use of wellestablished semiconductor technology enables the batch-production of arrays of hundreds of nanomechanical systems. Nanomechanical biosensors are usually cantilever-shaped. In comparison to quartz crystal resonators, the increasingly smaller size of nanomechanical systems is producing outstanding mass resolution on the verge of single atoms. In addition, nanomechanical systems can exhibit extremely low mechanical compliances translating 
biomolecular recognition events into measurable displacements. Since the size of the biomolecules is comparable to one of the dimensions of the mechanical system, mainly the thickness, the mechanical response is also highly sensitive to the mechanical properties of the adsorbed biomolecules, making this tool unique. Nanomechanical biosensors can be split into static and dynamic depending on whether the static displacement or the resonant properties of the nanomechanical system are measured (Fig. 3(a-b)). Several examples of nanomechanical systems used for biological detection that include cantilevers, doubly clamped beams and membranes are shown in Fig. 3(c-f).

(a)

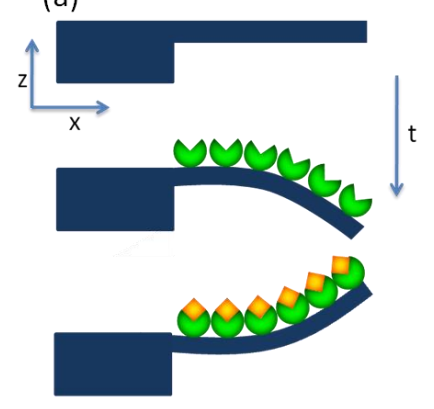

(b)
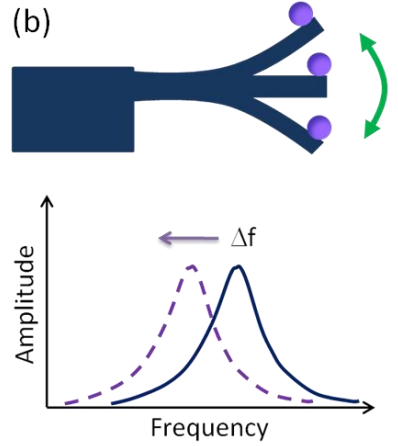

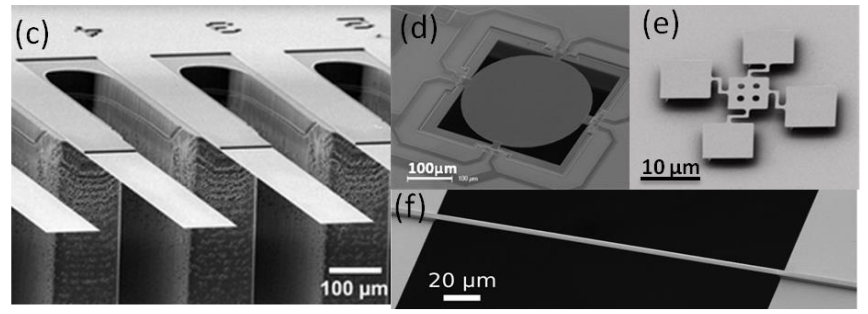

Figure 3. Main operation modes of nanomechanical biosensors: static mode (a) and dynamic mode (b). In the static mode, microcantilevers are commonly used. One side of the cantilever is functionalized to attach a monolayer of biomolecular receptors that exhibit high affinity to the targeted biomolecule. Molecular recognition induces changes in the surface stress of the upper side with respect to the lower that is mechanically relaxed by the cantilever bending. Displacement detection methods directly or indirectly measure the curvature change to derive the surface stress. In the dynamic mode, the cantilever is usually driven at its resonance frequency. Driving methods include piezoelectric actuators, magnetic forces, and light-induced forces, to name a few. When a biomolecule lands on the free end of a cantilever, the resonance frequency shifts downwards due to the added mass. Scanning electron microscopy images of different nanomechanical systems are shown: (c) microcantilever array (reprinted from ${ }^{18}$, (d) surface stress membrane-like sensor (used $\mathrm{in}^{36}$, kindly provided by Dr. Genki Yoshikawa), (e) microtrampoline (reprinted from ${ }^{37}$ ), (f) resonant nanostring (used in ref ${ }^{38}$, kindly provided by Dr. Silvan Schmid).

\subsubsection{Displacement detection techniques in nanomechanical biosensors}

Displacements in nanomechanical biosensors range from angstroms to hundreds of nanometers. These displacements can be measured by optical and electrical techniques with an exquisite sensitivity of at least $0.1 \mathrm{~nm} / \mathrm{Hz}^{1 / 2}$. We briefly review theses techniques. For further information, we refer the reader to several excellent reviews ${ }^{17,35}$. The most widely used method is the optical lever as it can be easily implemented in vacuum, air and liquids (the natural biological environment). The method consists in measuring the deflection of a laser beam reflected off the nanomechanical system surface by means of a position sensitive photodetector. Optical interferometric techniques can also be applied with similar 
performance. In addition, laser beam deflection and interferometric techniques have also been developed to image the displacement and vibration of the overall nanomechanical system, rather than just measuring the displacement at a single point. Figure 4 shows a schematic of the optical beam deflection technique ${ }^{39}$.

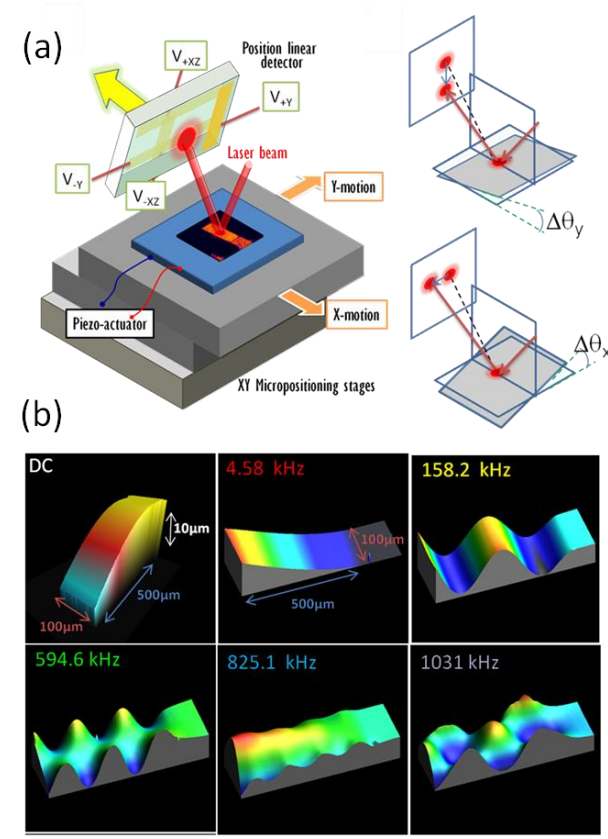

Figure 4. (a) Schematic representation of the scanning laser beam deflection system. A laser beam is tightly focused on the surface of the nanomechanical system. The reflected beam is collected on the surface of a two-dimensional position linear photodetector. Changes of the surface slope around the $\mathrm{Y}$-axis and $\mathrm{X}$-axis produce orthogonal displacements of the reflected beam on the position detector that can be independently measured. (b) Example of how by implementing scanning means and multifrequency excitation/detection, the laser beam deflection technique enables imaging of the static out-of-plane displacement and the shape of five vibration modes of a silicon microcantilever sensor The microcantilever is $500 \mu \mathrm{m}$ long, $100 \mu \mathrm{m}$ wide and $1 \mu \mathrm{m}$ thick.

Among electrical techniques, piezoresistive detection is the most widespread. This detection requires the integration of a piezoresisitive element during the fabrication of the nanomechanical system, which is usually located near the clamping regions. The change of resistance due to the motion-induced strain in the piezoelectric region is measured by a Wheatstone bridge ${ }^{17}$. The second most used electrical detection technique is the measurement of the capacity between the nanomechanical system and a parallel electrode ${ }^{35}$. Both electrical and optical detection techniques at the state of the art can achieve comparable displacement sensitivities in the range of $1-1000 \mathrm{fm} \mathrm{Hz}^{-1 / 2}$ and bandwidths of hundreds of $\mathrm{MHz}$. The electrical detection techniques impose higher complexity in the fabrication and major restrictions in the size, geometry and materials of the sensors. However, electrical detection provides an impressive degree of miniaturization which is crucial for point of care applications. Optical detection is bulky, thus more suited for bench-top equipment, but offers an enormous flexibility in the fabrication of nanomechanical systems that gives space for the 
development of novel concepts. For instance, the coupling between light and mechanics that occurs in optical cavities in which one of the mirrors is a flexible mechanical system opens the door for realizing novel optomechanical biosensors with enhanced performance ${ }^{40-42}$.

\subsection{Objectives of the review}

After this first glance at the field of nanomechanical biosensors, this review provides an insight on the mechanical phenomena that occurs in suspended mechanical structures when either biological adsorption or interactions take place on their surface. Based on these phenomena, we describe different kinds of nanomechanical biosensors developed so far, providing some examples of the most relevant reported applications. We discuss the intrinsic detection limits of the devices as well as the biological noise imposed by non-specific adsorption. Finally, we indicate possible future directions for this emerging field of research.

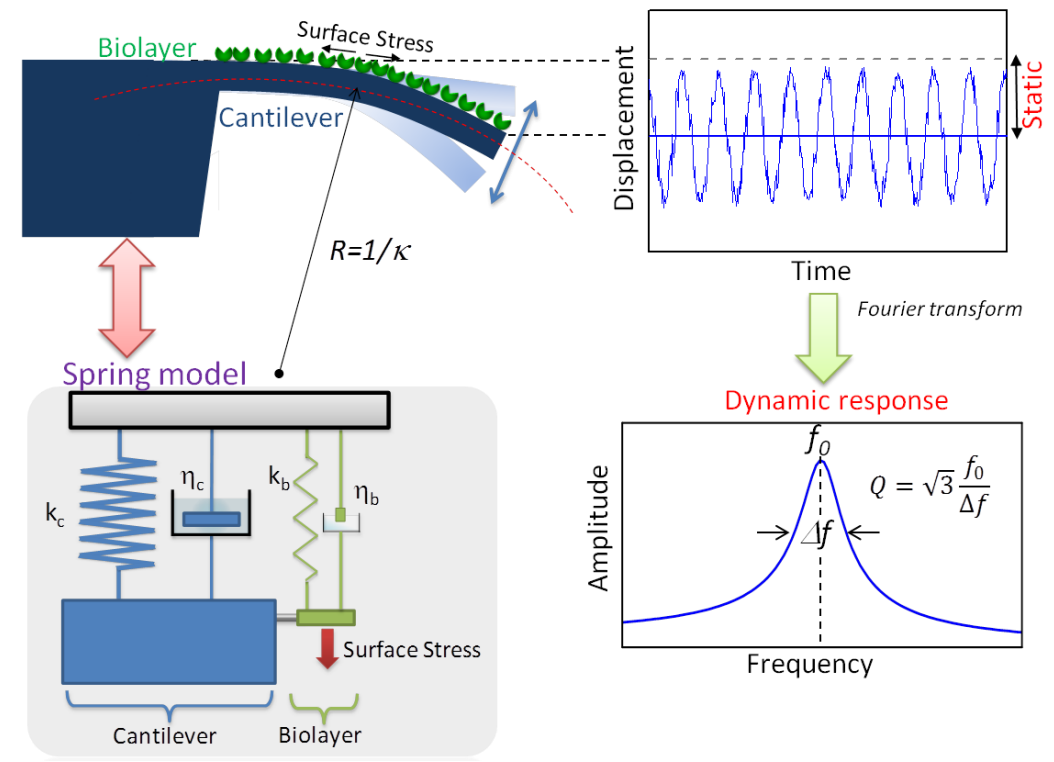

Figure 5. Schematic of the mechanical effects induced by biological adsorption on the response of a nanomechanical system, in this case, a microcantilever. The biolomolecules form a monolayer on the upper side of the microcantilever that generates surface stress producing static mechanical bending with curvature $\kappa$. In addition, the cantilever oscillates with respect to the equilibrium position. In this case, the oscillation is driven by thermal forces that are uncorrelated in time and space. The mean displacement signal arises from the surface stress induced cantilever bending, whereas the fluctuations can be analyzed in the frequency domain to determine the resonance frequency and quality factor $(Q)$. The equivalent spring model is useful to interpret the effect of the biological layers on the response of the cantilever. The elasticity and mechanical losses of the cantilever are modeled by a spring and a damper in parallel with spring constant $k_{c}$ and damping coefficient $\eta_{c}$, which are related to the flexural rigidity and quality factor of the cantilever, respectively. The biological layer acts as a spring and damper in parallel with a spring constant and damping coefficient $k_{b}$ and $\eta_{b}$, respectively. The biological layer brings about the increase of the mass, spring constant and damping coefficient of the overall system. Depending on the density and Young's modulus of the biological layer with respect to those of the cantilever, the resonance frequency can decrease or increase. In addition, the surface stress acts in the overall spring system by applying a force on the biological mass that is rigidly coupled to the cantilever mass giving rise to a displacement of the equilibrium position. 


\section{FUNDAMENTALS OF NANOMECHANICAL BIOSENSORS.}

We analyze now the changes that a nanomechanical system undergoes when biomolecules land on its surface. There are three mechanical quantities in addition to the added mass that change as a consequence of biomolecular adsorption: i) surface stress, ii) effective Young's modulus, and iii) viscoelasticity. All these changes will influence on the biosensor response that may include the following output signals (Fig. 5):

-Static bending of the nanomechanical system: generally produced by in-plane surface forces that generate changes of the curvature $(\kappa)$. The changes in the curvature can be monitored in real-time as the biomolecules interact with each other on the surface of the nanomechanical system. Typical averaging time in the displacement signal is of the order of a second.

-Resonance frequency (f): it depends on the geometry, size and clamping configuration. The flexural resonance frequencies of a beam with rectangular and uniform cross-section are given by $\mathrm{f}_{\mathrm{n}} \cong \frac{\beta_{\mathrm{n}}{ }^{2}}{21.7656}\left(\frac{\mathrm{E}}{\rho}\right)^{0.5} \frac{\mathrm{h}}{\mathrm{L}^{2}}$, where $E$ is the Young's modulus, $\rho$ is the material density, $h$ is the beam thickness, $L$ is the beam length and $\beta_{n}$ is the eigenvector. The first four eigenvectors for singly and doubly clamped beams are shown in Table I. The first eigenvector provides the fundamental resonance frequency, and the following values the resonance frequencies of the higher flexural vibration modes.

-Quality factor: it is defined by $Q=\frac{2 \pi W_{s}}{W_{d}}$, where $\mathrm{W}_{\mathrm{s}}$ is the stored vibrational energy and $\mathrm{W}_{\mathrm{d}}$ is the energy lost per cycle of vibration that can include thermoelastic losses, viscous damping and acoustic losses to the support. The quality factor significantly determines the accuracy of the measurement of the resonance frequency as it determines the slope of the amplitude and phase curves near resonance. Thus, $\mathrm{Q}$ is also defined by, $Q=\sqrt{3} \frac{f_{0}}{\Delta f}$ where $\Delta f$ is the resonant peak frequency full-width at half-maximum (see figure 5).

TABLE I. Numerical solutions for a singly and doubly clamped beams.

\begin{tabular}{|c|c|c|c|c|}
\hline \multicolumn{5}{|c|}{ SINGLY CLAMPED BEAMS } \\
\hline & $n=1$ & 2 & 3 & 4 \\
\hline$\beta_{n}$ & 1.8751 & 4.69409 & 7.85476 & 10.9955 \\
\hline$C_{1 n}$ & 1.00000 & 1.00000 & 1.00000 & 1 \\
\hline$C_{2 n}$ & 0.734096 & 1.01847 & 0.999224 & 1.00003 \\
\hline \multicolumn{5}{|c|}{ DOUBLY CLAMPED BEAMS } \\
\hline & $n=1$ & 2 & 3 & 4 \\
\hline$\beta_{n}$ & 4.73004 & 7.8532 & 10.9956 & 14.1372 \\
\hline$C_{1 n}$ & 1.00000 & 1.00000 & 1.00000 & 0.999997 \\
\hline$C_{2 n}$ & 0.982502 & 1.00078 & 0.999966 & 0.999998 \\
\hline
\end{tabular}


In Fig. 6, we show the main mechanical structures used in this review: a singly clamped beam (cantilever) and a doubly clamped beam. The length, width and thickness are referred to as $L, b$, and $h$, respectively. The beams are oriented along the $x$ axis, with displacements along the $z$ axis. This nomenclature and the coordinate system shown in Fig. 6 will be used in this review hereinafter.

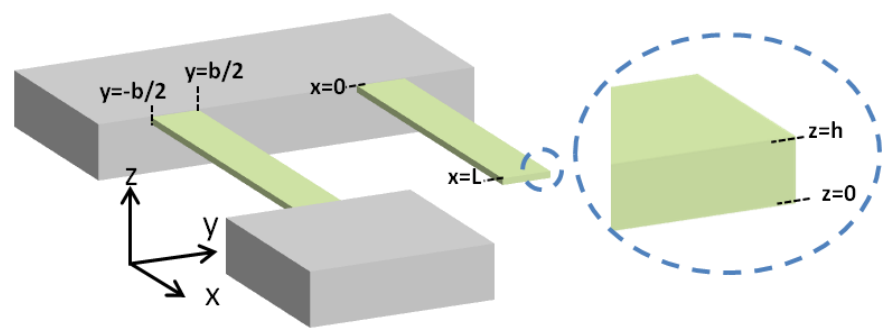

Figure 6. Schematic diagram of singly and doubly clamped beams with length $L$, width $b$ and thickness $h$. The end supports are assumed infinitely rigid.

\subsection{Surface stress effect on nanomechanical systems}

Surface stress is the amount of reversible work per unit area needed to elastically stretch a pre-existing surface. Adsorption on a surface can generate changes in the surface stress as a consequence of the adsorbate/surface and adsorbate/adsorbate interactions ${ }^{43-45}$.

\subsubsection{Static nanomechanical response to surface stress}

Molecular recognition induces surface stress on one side of a nanomechanical system (active surface) and gives rise to the bending of the system ${ }^{19,44-45}$ (Fig. 7). The bending arises from the surface energy variation of the active surface that is minimized by elastic expansion or contraction with respect to the opposite surface (passive surface). This effect has been the basis of a wide number of label-free transduction of ligand-receptor interactions such as nucleic acid hybridization, immunoreactions, carbohydrate-protein interactions and antibiotic/bacteria interactions as summarized below ${ }^{14-20,46-54}$. Cantilevers have been the dominant geometry for biological sensing based on the surface stress due to their low spring constant. Recently, a membrane-type surface stress sensor suspended by four piezoresistive "sensing beams", has been proposed with enhanced sensitivity in comparison to cantilevers ${ }^{36}$ (Fig. 3(d)). The surface stress variation is obtained from the measurement of the change of curvature by applying Stoney's equation which provides a linear relationship between surface stress change $(\Delta s)$ and the curvature change $(\Delta \kappa)^{55}$,

$\Delta \kappa=6 \frac{1-v}{E h^{2}} \Delta s$ 
where $E$ is the Young's modulus, $v$ is the Poisson's ratio and $h$ is the beam thickness. Equation (1) assumes that the beam is isotropic and homogeneous, the beam thickness is small compared to the lateral dimensions, and the strains and rotations are small (the effect of inplane stress on the out-of-plane displacement is negligible). This adequately complies with the most commonly used microcantilevers with length, width and thickness that typically fall in the range 100-500 $\mu \mathrm{m}, 10-100 \mu \mathrm{m}, 0.1-1 \mu \mathrm{m}$, respectively. Stoney's equation was published in $1909^{56}$ as a result of the observations of how glass substrates bent when metals were deposited on them. This equation is only strictly valid for plates unrestrained along the edges that exhibit uniform curvature (Fig. 7(a)). Thus, equation (1) can induce significant errors in measurements with microcantilevers in which one edge is rigidly clamped to a chip substrate ${ }^{55}$ (Fig. 7(b)). Recently, a simpler equation has been derived that accurately quantifies the clamping effect, and provides the curvature averaged across the beam width as a function of the longitudinal position $(x)$,

$\overline{\Delta \kappa}(x)=\Delta \kappa_{S t}\left(1+v e^{-\alpha \frac{x}{b}}\right)$

where $\Delta \kappa_{s t}$ is the Stoney's curvature (eq. (1)), $\alpha(v) \cong 2.2803+1.0056 v-0.1762 v^{2}$. The obtained solution indicates that the clamping restriction induces an additional curvature to the uniform curvature derived by Stoney. This term exponentially decays in the longitudinal direction with a characteristic length scale that is linearly proportional to the cantilever's width and to a constant that weakly varies from 0.44 to 0.37 when the Poisson's ratio increases from 0 to 0.5 .

(a)

(b)
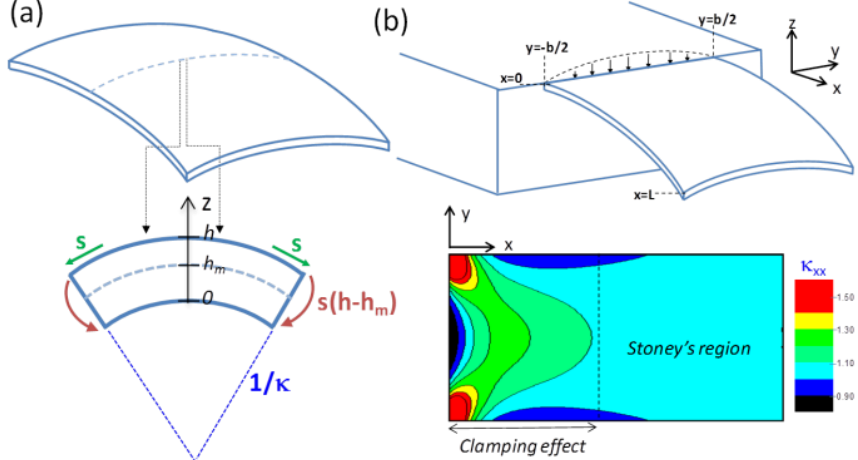

Figure 7. (a) Schematic of the bending of an unrestrained rectangular plate when surface stress is developed in the upper face. The bottom diagram shows the surface forces, $s$, and bending moments, $s\left(h-h_{m}\right)$ exerted on a crosssection element of the plate, where $h_{m}$ is the neutral axis position. The plate bends with uniform curvature $\kappa$. (b) Schematic of the bending of the rectangular plate shown in (a) when it is clamped to a substrate. The clamping exerts reaction forces to cancel out the curvature induced by the bending moment. This results into a non uniform curvature as shown in the finite element simulations of the curvature in $x\left(\kappa_{x x}\right)$ shown below for a cantilever with an aspect ratio $L / b=2$. The simulation shows that the clamping induces a curvature that decays in the $x$ direction with a characteristic length scale given by the cantilever width $b$. The cantilever region where $x>b$ approximately exhibits a uniform curvature that obeys Stoney's equation. 
The equations described above assume a planar geometry. Real surfaces, however, exhibit corrugation at atomic, nanometer, and micrometer scales. In terms of geometry, roughness increases the surface area that should contribute to a higher sensitivity, and lets the surface forces act at an angle to the substrate plane, which should decrease the sensitivity ${ }^{57-59}$. The effect of the roughness on the surface stress induced bending curvature is still controversial. It has been reported that for the case of random roughness, the cantilever sensitivity is found to significantly decrease with increasing roughness or equivalently increasing local surface slope ${ }^{59}$. The effect is amplified by Poisson's ratio. In contrast, it has been recently reported a combination of atomistic simulations and a simple continuum model that implies that surface stress is negligibly impacted by surface roughness ${ }^{58}$. However, the authors discovered that even a moderate roughness is seen to dramatically alter the surface elasticity modulus (generally ignored), which could explain the discrepancies.

\subsubsection{Surface stress effect on resonance frequency}

Surface stress changes can also influence on the resonance frequency of nanomechanical systems. This effect depends on the nanomechanical system geometry. In doubly clamped beams, the surface stress induces a force along the beam axis that modifies the resonance frequency. This effect is analog to the vibration of a guitar string under tension, and can be theoretically predicted within the framework of Euler-Bernoulli beam theory ${ }^{60-61}$. In contrast, the effect of surface stress on the resonance frequency of cantilever beams has remained an unsolved problem in physical sciences during the last three decades that has originated controversial experimental and theoretical reports ${ }^{62-65}$. Recently, several studies have solved this issue. Previous models simplified the problem by replacing the surface stress with an external axial force integrated over the cantilever dimensions ${ }^{62,65}$. However, the axial force model that is valid for doubly clamped beam is unsatisfactory for singly clamped beams. The reason is simple, a cantilever has a free end that allows deformation to relieve the stress, so

the frequency should not change ${ }^{63-64}$. However, this one dimensional view of the problem does not capture the effect of the clamping. When the stress and strains exerted on a cantilever plate are analyzed in the two dimensions, it is noted that the in-plane-forces near the clamp cannot be relieved. The clamping restriction induces nonzero in-plane stresses that decay in the direction along the cantilever beam with a characteristic length scale given by the cantilever width $b^{60-61,66}$. This effect generally induces frequency shifts of the order of $100 \mathrm{ppm}$. A second effect of surface stress is the elastic change in the nanomechanical lateral dimensions. This "geometric" effect is typically ignored in the classical theory of linear elasticity, but it may be comparable to the effect of in-plane stress near the clamping ${ }^{61}$. The 
effect of surface stress on the resonance frequencies of singly and doubly clamped beams are respectively given by,

$\frac{\Delta f}{f_{0}} \cong \frac{1-v}{E h}\left[-0.042 v \frac{b^{3}}{L h^{2}}+\frac{1+2 v}{1-v}\right]\left(s_{u}+s_{b}\right)$

$\frac{\Delta f}{f_{0}} \cong \frac{1-v}{E h}\left[0.1475\left(\frac{L}{h}\right)^{2}+\frac{(1+2 v)(1+v)}{2(1-v)}\right]\left(s_{u}+s_{b}\right)$

where the second summand arises from the geometric effect and the first summand from: (i) in-plane stresses near the clamp in cantilevers, and (ii) a net axial force in doubly clamped beams; $s_{u}$ and $s_{b}$ are the surface stress in the upper and bottom sides of the beam. The formulas are derived under the assumption of a thin beam $(\mathrm{h}<<\mathrm{b})$, where the stress load is applied to an infinitesimal layer at the surface.

The surface stress effect described above is derived in the framework of linear beam deformation. In this situation, the cantilever displacements are assumed to be small with regards to the cantilever thickness. However, this assumption is extremely restrictive from the experimental point of view where large deflections are sought for small surface stress variations. This is achieved by using ultrathin microcantilevers with large aspect-ratio. In these cases, non-linear effects can be much higher than the linear effects ${ }^{67}$. Non-linear effects arise from the large beam bending induced by differential surface stress between the opposite sides of the nanomechanical systems $\left(s_{u} \neq s_{b}\right)$, In this situation, the coupling between in-plane and out of plane strains is sufficiently high to give rise to an effective "softening" or "hardening" of the cantilever. This effect has been revealed as a bistable dyanamic behavior of cantilevers at high amplitudes ${ }^{68}$. We have recently calculated the non-linear effect of differential surface stress between on the resonance frequency of cantilevers with high aspect ratios ${ }^{69}$,

$$
\frac{\Delta f}{f_{0}} \cong 0.7\left[(1-v) \frac{L\left(s_{u}-s_{b}\right)}{E h^{2}}\right]^{2}
$$

In contrast with the linear effect of surface stress, the sign of the resonance frequency shift is independent of the sign of differential surface stress.

\subsection{Mass effect on nanomechanical systems}

\subsubsection{Mass effect on resonance frequency}


A nanomechanical system set into oscillation at its natural frequency functions as precision mass sensors, like its "big brother", the quartz crystal microbalance. Nanomechanical systems exploit the miniaturization roadmap in microelectronics taking advantage of the most recent developments in micro- and nanofabrication technologies. Thus, as the physical size is reduced, the mass sensitivity of nanomechanical resonators is enhanced, being inversely proportional to the mass of the devices. Resonators fabricated by top-down technologies have proven a detection limit in the zeptogram range $\left(10^{-21} \mathrm{~g}\right)$, and nanomechanical sensors assembled from bottom-up synthesized nanostructures, such as semiconductor nanowires (NWs) or carbon nanotubes (NTs), have even approached to the yoctogram scale $\left(10^{-24} \mathrm{~g}\right)$. Notice that the mass of one single hydrogen atom is 1.66 yoctogram. Figure 8 summarizes these achievements in mass detection ${ }^{70-75}$. The plotted data represent measurements in vacuum of deposited masses consisting of atoms or molecules. The measurements in vacuum are crucial to achieve the highest sensitivity as the mechanical resonators exhibit the highest quality factor possible (1000-10 000).

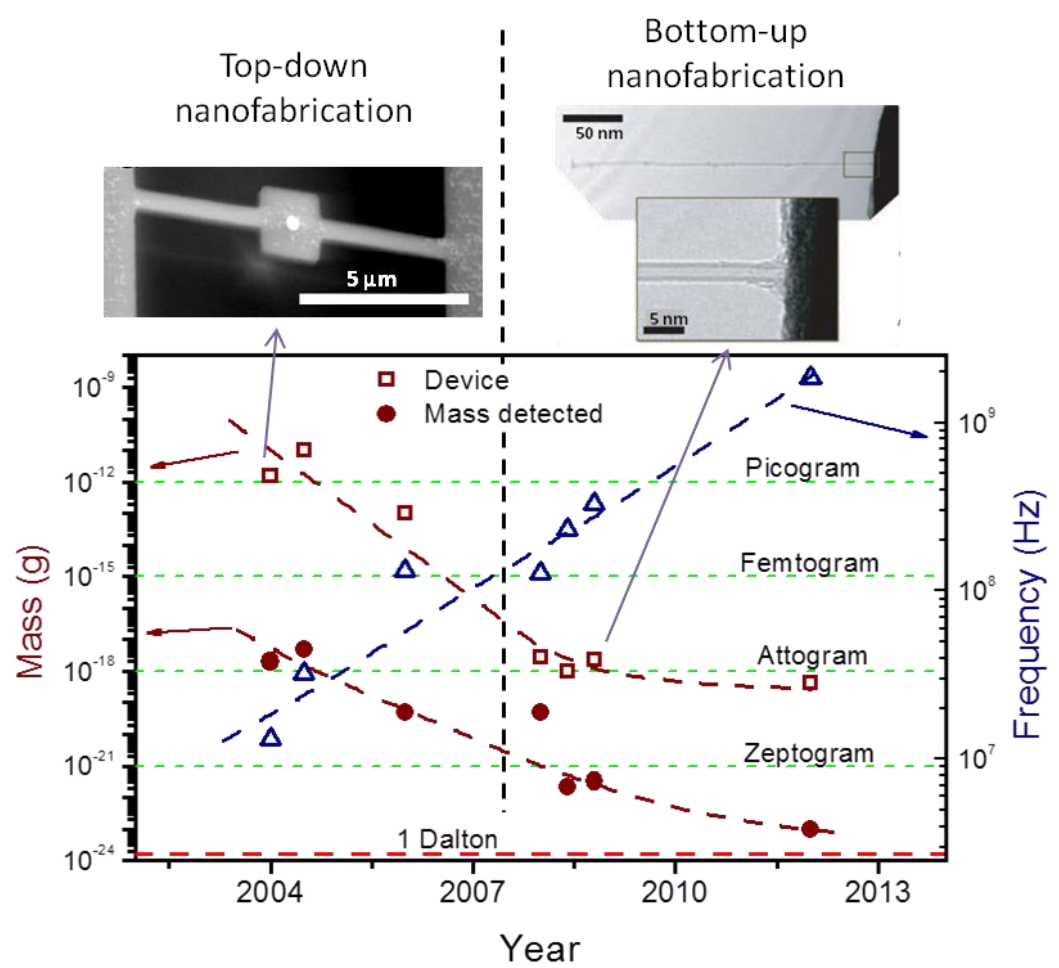

Figure 8. Chronology of the advances in nanofabrication of mechanical resonators that have increasingly enhanced the mass detection limits. The data are from references ${ }^{70-75}$. The device mass, the detected mass levels and the resonance frequencies are plotted. All the data represent measurements in vacuum, in which the quality factor is high (1000-10 000) and the resonance frequency can be measured with high sensitivity. The masses are atoms or molecules $\left(\mathrm{Au}, \mathrm{Cr}, \mathrm{Xe}, \mathrm{C}_{10} \mathrm{H}_{8}\right)$ deposited in the measurement vacuum chamber, except for the first data point in which the added mass arises from immersion in a solution containing thiolated molecules to achieve their binding on a gold nanodot fabricated in the active part of the mechanical resonator (see image at left top corner) ${ }^{70}$. In this case, the measurement is performed in vacuum before and after the incubation. The advances in nanofabrication have led to smaller devices, smaller mass detection limits at a logarithmic approaching Dalton resolution. Consequently, the resonance frequencies have increased at a logarithmic rate. Up to 2006, the nanofabrication was mainly performed by top-down techniques based on lithography, such as e-beam nanolithography. After 2006, resonators based on carbon nanotubes and silicon nanowires were used to achieve higher detection limits. 
For the case of a biological particle adsorbed on a nanomechanical resonator, the mass effect on the resonance frequency strongly depends on the adsorption position and the used vibration mode. The resonance frequency shift $(\Delta f)$ can be mathematically expressed by the approximate simple equation,

$\frac{\Delta f_{n}}{f_{0 n}}=-\frac{1}{2} \psi_{n}^{2}(x, y) \frac{m_{a}}{m_{b}}$

where $m_{a}$ is the mass of the biological particle, $m_{b}$ is the beam mass, $f_{o n}$ is the resonant frequency of the unloaded resonator for $n^{\text {th }}$ vibration mode, $\psi_{n}$ is the amplitude vibration mode shape of the $n^{\text {th }}$ vibration mode (also referred to as eigenfunctions) and $(x, y)$ are the adsorption coordinates. The index $\mathrm{n}$ ranges from zero (fundamental mode) to infinity (notice that only a limited number of vibration modes can be observed as the amplitude for a given excitation force severely decreases with the frequency). The amplitude of the flexural vibration modes (that are the most used) for singly and doubly clamped beams are given by,

$\psi_{n}(\zeta)=C_{1 n} \operatorname{Cos}\left(\beta_{n} \zeta\right)-C_{1 n} \operatorname{Cosh}\left(\beta_{n} \zeta\right)-C_{2 n} \operatorname{Sin}\left(\beta_{n} \zeta\right)+C_{2 n} \operatorname{Sinh}\left(\beta_{n} \zeta\right)$

where $\zeta$ is the $x$-coordinate normalized by the beam length. The eigenfunctions are normalized so that $\int_{0}^{1} \psi_{n}(\zeta)^{2} d \zeta=1$. The eigenvector $\beta_{n}$ and constants $C_{1 n}$ and $C_{2 n}$ are summarized in Table I.

The follow up of the effect of a biological particle on a nanomechanical resonator is practical for biological nanomechanical spectrometers in which individual particles sequentially attach to the surface of the resonator, as shown below. In other instruments such as affinitybased biosensors, the analytes are uniformly distributed across the beam. In this case, the resonance frequency is given by,

$\frac{\Delta f_{n}}{f_{0 n}} \cong-\frac{1}{2} \frac{\mathrm{m}_{\mathrm{a}}}{\mathrm{m}_{\mathrm{b}}}$

\subsubsection{Mass effect on quality factor}

The quality factor provides another source of information on the adsorbate mass. For pressures above $10^{-2} \mathrm{mbar}$, the quality factor of a beam is determined by the surrounding medium. In addition, the effect of the medium on both the resonance frequency and quality factor is known ${ }^{76-77}$. This knowledge leads to that the effect of the mass on the quality factor of the $\mathrm{n}^{\text {th }}$ vibration mode $\left(Q_{n}\right)$ for the case of homogeneous adsorption is given by ${ }^{78}$, 
$\frac{Q_{n}}{Q_{0 n}}=\left(1+\frac{\mathrm{m}_{\mathrm{a}}}{\mathrm{m}_{\mathrm{b}}}\right) \frac{\operatorname{Im} \Gamma\left(f_{n}\right)}{\operatorname{Im} \Gamma\left(f_{0 n}\right)}$

where $Q_{0 n}$ is the unloaded quality factor and $\Gamma$ is the known hydrodynamic function for a rectangular beam, which depends on the frequency and the width of the cantilever, and on the density and the viscosity of the medium ${ }^{76-77}$. Equation (8) is only strictly valid for $Q_{0 n}>>1$.

\subsection{Stiffness effect on nanomechanical systems}

\subsubsection{Stiffness effect on the static bending}

When surface stress biosensors make use of ultrathin nanomechanical devices, the thickness of the biological layer is comparable to that of the device. Modeling of these systems requires the lifting of the thin film approximation. Thus, the elastic properties of the biomolecules anchored to the nanomechanical system can significantly modify the bending responsivity to surface stress changes ${ }^{79}$. This effect must be accounted for and can be combined with other measurements to ascertain the elasticity of biological layers that are receiving an increasing interest in biology.

\subsubsection{Stiffness effect on the resonant frequencies}

Until 2006, sensors based on nanomechanical resonators were often referred to as resonant mass sensors. Then, it was reported the surprising fact that soft matter such as a cluster of bacteria cells can induce a positive resonance frequency shift in microcantilevers for

certain adsorption distributions over the cantilever ${ }^{80-82}$ (Figure 9). These results are not so unexpected when one considers the energies involved in the oscillations of nanomechanical resonators $^{83}$. Let us consider a beam that oscillates with a transverse amplitude $u(x, t)=$ $A \psi_{n}(\mathrm{x}) \cos \left(2 \pi f_{n} t+\phi\right)$, where $\mathrm{A}$ and $\phi$ are arbitrary values of the amplitude and phase. The mean values of the beam bending work and kinetic energy per oscillation cycle are respectively given by

$\left\langle W_{b}\right\rangle=\frac{A^{2}}{4 L^{3}} \int_{0}^{1} D(\zeta) \frac{d^{2} \psi_{n}(\zeta)^{2}}{d \zeta^{2}} d \zeta$

$\left\langle W_{k}\right\rangle=A^{2} \pi^{2} L f_{n}^{2} \int_{0}^{1} \rho(\zeta) \psi_{n}(\zeta)^{2} d \zeta$

where $\rho$ is the cantilever mass density, and $D$ is the flexural rigidity of the cantilever defined as the ratio between bending moment and the curvature. For uniform beams $D=E l$ where $I$ is the second moment of inertia that for a rectangular cross-section is $l=b h^{3} / 12$. 
In equilibrium, the average rates of kinetic energy and potential energy must equal. This equality provides the resonance frequency and explains why when a mass is added to a resonator the resonance frequency decreases due to the kinetic energy increase. In a similar way, the mechanical stiffness of the adsorbate implies a potential energy cost during the beam bending that induces a positive resonance frequency. The mass effect increases when adsorption takes place at regions along the beam with high amplitude (higher kinetic energy), whereas the stiffness effect increases in the regions where the beam undergoes higher changes of curvature during vibration (higher potential energy). An extended equation for the resonance frequency shift due to the adsorption of a particle on a beam that accounts in addition to the mass effect, for the stiffness effect is ${ }^{83}$ :

$\frac{\Delta f_{n}}{f_{0 n}}=\left(\frac{3}{2} \frac{1}{\beta_{n}{ }^{4}} \frac{d^{2} \psi_{n}(\zeta)^{2}}{d \zeta^{2}} \frac{\mathrm{E}_{\mathrm{a}}}{\mathrm{E}_{\mathrm{b}}}-\frac{1}{2} \psi_{n}^{2}(\zeta) \frac{\rho_{\mathrm{a}}}{\rho_{\mathrm{b}}}\right) \frac{\mathrm{V}_{\mathrm{a}}}{\mathrm{V}_{\mathrm{b}}}$

where $V$ is volume and the subscripts $a$ and $b$ denote the beam and adsorbate materials, respectively.

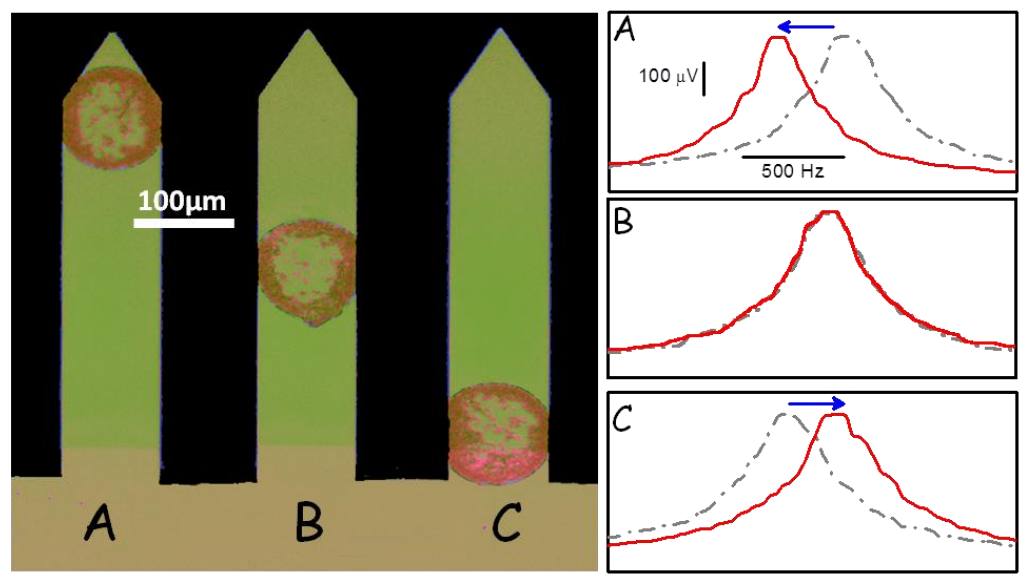

Figure 9. Left: optical micrograph of three silicon cantilevers labeled as A, B and C, in which bacterial cells have been deposited by inkjet at three different positions along the cantilever length. The cantilevers are $500 \mu \mathrm{m}$ long, $100 \mu \mathrm{m}$ wide, and $1 \mu \mathrm{m}$ thick. The center of the bacteria spot is separated from the clamping 73, 200, and $390 \mu \mathrm{m}$ in cantilevers A, B and C, respectively. Right: frequency spectra of the Brownian motion of the three cantilevers shown at the left, before (grey discontinuous line) and after (continuous red line) bacteria deposition. The measurements were performed in air. The shown frequency span allows the visualization of the first resonance mode located between 7.0 and $8.5 \mathrm{kHz}$. 
Figure 10 shows the resonance frequency responsivity due to the stiffness effect (first summand in eq. (10)) and to the mass effect (second summand in eq. (10)) for the first two vibration modes. The responsivities for the mass and stiffness effects are proportional to square of the amplitude and curvature of the vibration mode shape at the adsorption site, respectively. For the fundamental vibration mode of singly clamped beams, the regions of highest amplitude/null curvature and highest curvature/null amplitude are located near the free and fixed ends of the beam. Thus, the mass and stiffness of the adsorbate can correspondingly be uncoupled by measuring the resonance frequency shift induced by the particle adsorption at the free and fixed ends of a beam, respectively. In the fundamental vibration mode of doubly clamped beams, the region of highest amplitude is also a region of high curvature, so the frequency shift due to a particle in the middle of the beam has a positive component due to the stiffness and a negative component due to the mass. These counteracting effects can be detrimental for the sensitivity. However, adsorption near the clamp only induces a positive stiffness-induced frequency shift. Following the same reasoning for the higher vibration modes, in singly clamped beams, the stiffness effect is dominant in regions near the clamp and around the vibration nodes (regions of null vibration and high curvature), whereas the mass is only dominant when the particle adsorbs near the free end. For doubly clamped beams, the stiffness effect is dominant for adsorption near the clamps and vibration nodes, but there is no adsorption region where only the added mass influences on the frequency shift.

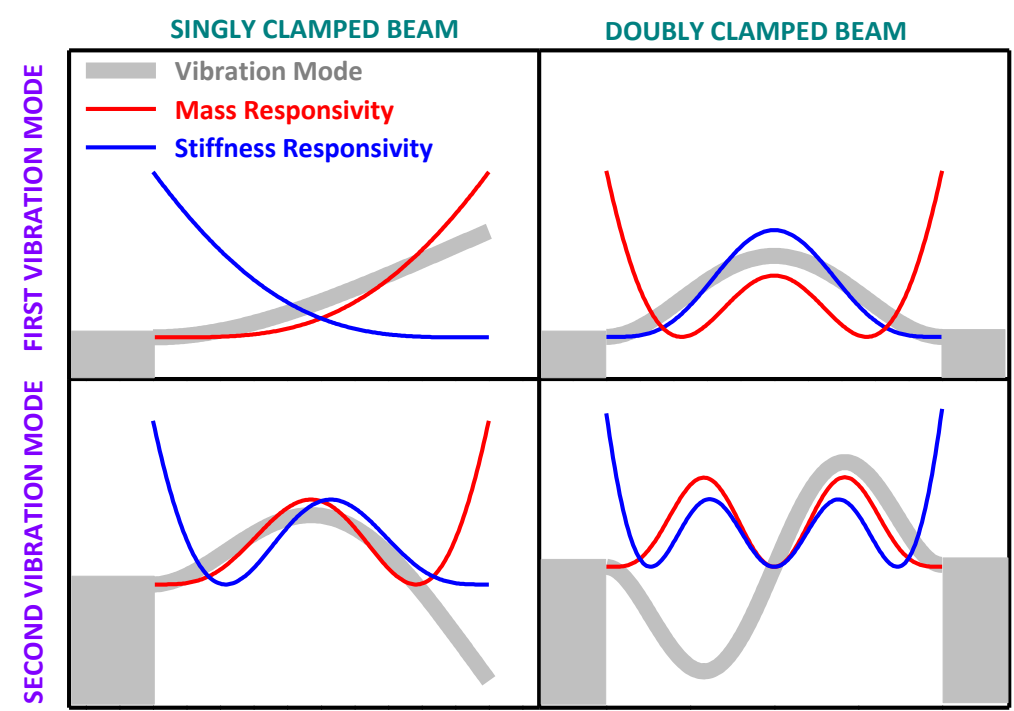

Figure 10. Resonance frequency responsivity to punctual changes of mass (red line) and stiffness (blue line) for singly and doubly clamped beams for the first two vibration modes. The shapes of the vibration modes are also plotted (grey thick line) together a sketch of the chip to which the beams are clamped. The responsivities for the mass and stiffness effects are proportional to the square of the amplitude and curvature of the vibration shape, respectively. 
In order to get a flavor of how the mechanical and mass properties of a biological system influence on the resonance frequency response of nanomechanical resonators, we have calculated the shift of the fundamental resonance frequency for a viral particle in a singly and a doubly clamped beams with length $L=5 \mu \mathrm{m}$, width $\mathrm{b}=0.5 \mu \mathrm{m}$ and thickness $\mathrm{h}=0.05 \mu \mathrm{m}$ (Fig. 11). We have used eq. (10). The chosen virus is the tobacco mosaic virus (TMV) that is a hollow tubular structure formed by the self-assembly of 2130 identical protein subunits (molecular weight $17.5 \mathrm{kDa}$ ) in a right-handed helical motif around a single-strand genomic RNA which forms a hairpin loop structure ${ }^{84}$. It has a length of $250 \mathrm{~nm}$ and outer and inner diameters of 18 and $4 \mathrm{~nm}$, respectively. The axial Young's modulus of TMV nanotubes estimated by atomic force microscopy bending measurements is of about $6 \mathrm{GPa}^{85}$. The resonance frequency shifts are calculated for two beam materials widely used in micro- and nanofabrication, silicon $\left(\rho_{b}=2330 \mathrm{~kg} / \mathrm{m}^{3}, E_{b}=169 \mathrm{GPa}\right)$ and the photosensitive polymer SU8 $\left(\rho_{b}=1190 \mathrm{~kg} / \mathrm{m}^{3}, E_{b}=4 \mathrm{GPa}\right)^{86}$. As described above, for singly clamped beams the mass and stiffness properties of the virus are uncoupled when the viral particle adsorbs near the free end or near the clamp, respectively. However, as the chosen beam material is softer, as it is the case of SU8, the mechanical properties of the virus play a predominant role. In doubly clamped beams, the mass of the virus cannot directly be inferred as there is always a stiffness contribution. This is more noticeable for the SU8 beam.

(a)

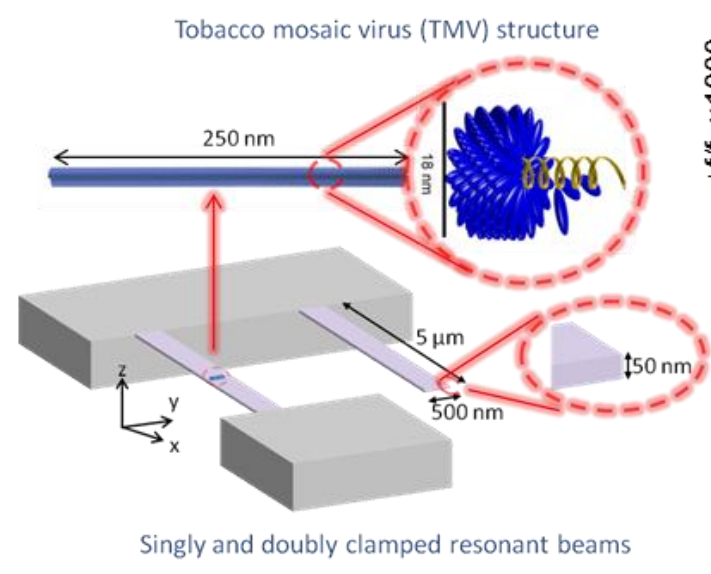

(b)

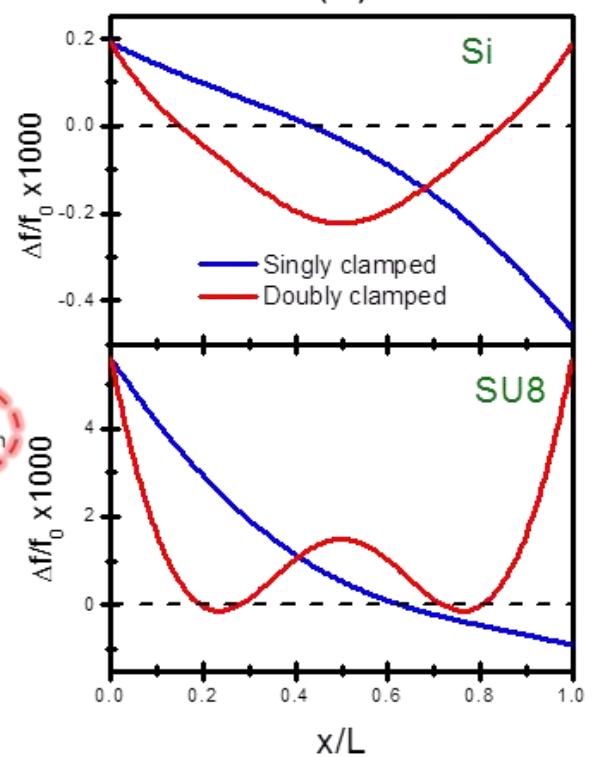

Figure 11. (a) Schematic depiction of the adsorption of the tobacco mosaic virus in a singly and a doubly clamped beams. The sizes and geometries of the beam and virus are shown. (b) Calculation of the relative shift of the resonance frequency of the fundamental vibration mode for SU8 and Si beams upon adsorption of the virus at position $\mathrm{x}$ normalized to the beam length $\mathrm{L}$. 
By integration of equation (10), we obtain the resonance frequency shift for the case of homogeneous adsorption, in which an effective layer of thickness $h_{a}$ is formed on the beam,

$\frac{\Delta f_{n}}{f_{0 n}} \cong\left(\frac{3}{2} \frac{E_{a}}{E_{\mathrm{b}}}-\frac{1}{2} \frac{\rho_{\mathrm{a}}}{\rho_{\mathrm{b}}}\right) \frac{\mathrm{h}_{\mathrm{a}}}{\mathrm{h}_{\mathrm{b}}}+\frac{3}{8}\left[\left(\frac{\rho_{\mathrm{a}}}{\rho_{\mathrm{b}}}\right)^{2}-3\left(\frac{\mathrm{E}_{\mathrm{a}}}{\mathrm{E}_{\mathrm{b}}}\right)^{2}-2 \frac{\mathrm{E}_{\mathrm{a}}}{\mathrm{E}_{\mathrm{b}}} \frac{\rho_{\mathrm{a}}}{\rho_{\mathrm{b}}}\right]\left(\frac{\mathrm{h}_{\mathrm{a}}}{\mathrm{h}_{\mathrm{b}}}\right)^{2}$

\subsection{Viscoelasticity effects on nanomechanical systems.}

Quartz crystal microbalance biosensors have been developed in the last decade to obtain quantitative and sensitive information on the viscoelastic properties of biological films in liquids ${ }^{87-88}$. The key element in these experiments is the simultaneous measurement of the resonance frequency $(5-25 \mathrm{MHz})$ and quality factor that is high in liquids $(\sim 1000)$ due to the shear wave based vibration. The relevance of the viscoelastic properties of a biofilm lies on that the biofilm cannot be regarded as a "dead mass", the effective coupled mass depends on how the vibration of the resonator propagates to the adsorbed film. A second aspect of resonance measurements in liquids is that water molecules may couple as an additional mass via direct hydration, viscous drag, or entrapment in cavities in the adsorbed film. This means that the biological layer is essentially sensed as a viscoelastic "hydrogel" composed of the macromolecules and the coupled water. In experiments of protein adsorption on quartz crystal microbalances, quantitative data about the thickness, shear elastic modulus, and shear viscosity of the protein film have been obtained by measuring the resonance frequency and quality factor, and by modeling the protein film as a Voigt viscoelastic system.

Quartz crystal microbalances are large scale devices with diameters of typically $14 \mathrm{~mm}$. The use of nanomechanical resonators with sizes of 10-100 $\mu \mathrm{m}$ would enable measuring precious biological samples with much higher sensitivity. Unfortunately, the measurements of viscoelastic properties with nanomechanical resonators are scarce ${ }^{89-90}$. The reason is that the quality factor of flexural resonators (microcantilevers and doubly clamped beams) is very low in liquids, generally below 10 , as a consequence of the severe viscous damping. Notice that the sensitivity to measure energy losses is proportional to the quality factor. Unfortunately, miniaturization of the resonators to the nanoscale does not help, and may worsen the quality factors ${ }^{91-92}$. Researchers are trying to "catch" the concept of quartz crystal microbalances in which the viscous dissipation is largely minimized when the surface oscillates parallel to the liquid/solid interface instead of transversally as it occurs in singly and doubly clamped beams when they oscillate in flexural modes ${ }^{93-95}$. Recently, advances in micro- and nanofabrication have provided micromechanical resonators based on the extensional vibration modes for a 
variety of mechanical geometries with frequencies of tens of $\mathrm{MHz}$ and quality factors in liquids of $1000-2000^{93}$. These devices hold promise for measuring viscoelastic properties of biological systems with unprecedented sensitivity.

\section{IMMOBILIZATION OF BIOLOGICAL RECEPTORS}

To turn a nanomechanical system into a biosensor, it must be coated with a biorecognition element, usually a layer of biomolecular receptors such as nucleic acids, proteins and carbohydrates. The functionalization of the cantilever surface comprises (i) the chemical modification of the surface to obtain reactive functional groups that strongly bind the biorecognition element, (ii) the immobilization of the biorecognition element and, finally, (iii) if bare areas remain on the cantilever surface, they must be blocked with anti-fouling molecules to minimize non-specific adsorption. In common with any biosensor, the immobilization of the biomolecular receptors must ensure the natural activity of the receptors, its bioavailability to targets and the formation of stable, robust, homogeneous and reproducible sensing layers. The functionalization of nanomechanical systems shares many aspects with the functionalization techniques of biosensors in general. Thus, we recommend to the readers interested in biofunctionalization of surfaces some of the reviews of the extensive literature in the subject ${ }^{96}$. The distinctive requirements that biofunctionalization methods of nanomechanical systems must satisfy are:

i) Nanomechanical systems are fragile, hence mechanical agitation for mixing and cleaning must be gentle.

ii) Some fabrication procedures such as surface micromachining provides nanomechanical systems separated from the substrate hundreds of nanometers (the thickness of the sacrificial layer used in the fabrication) ${ }^{15,20}$. During functionalization, stiction may occur, which leaves the device non operational. An obvious approach to avoid stiction is to increase both the effective spring constant of device and the distance between the device and the substrate. Alternatively, in devices prone to stiction, critical point drying procedures can be used ${ }^{20}$. The problem here is to preserve the biological activity of the receptors on the device surface.

iii) In the case of electrical displacement transduction, special care must be taken to avoid wetting of the electrical contacts during functionalization. This is usually avoided by isolation of the contacts with electrical insulating materials such as silicon nitride and biocompatible polymers ${ }^{15}$. 
iv)To capitalize the small size of the nanomechanical devices, methods for delivery ultralow volumes of samples and reagents with high spatial resolution are required.

We now review the most widely employed techniques for biological functionalization of nanomechanical systems.

\subsection{Immobilization on gold surfaces}

Gold is one of the most used surfaces for immobilizing bioreceptors on nanomechanical systems, especially in cantilever-based biosensors that measure the bending due to surface stress variations between opposite cantilever sides. In this case, the required asymmetry in the coating is achieved by depositing a 20-30 nm thick gold layer on one of the cantilever sides by thermal, e-beam or sputtering deposition methods. A 2-5 nm thick intermediate layer of chromium or titanium is used to enhance the adhesion between the gold and the cantilever surface. The high affinity of sulfur for gold is harnessed to form self-assembled monolayers (SAMs) of molecules derivatized with a thiol group (-SH) with useful chemical or biological functionalities at the coated cantilever surface ${ }^{97-98}$. For instance, nucleic acid probes can be synthesized with thiol linkers ${ }^{46-47,49,94,99-101}$ and proteins can be attached to SAMs with active groups that strongly bind primary aminoacid residues ${ }^{51-52,102}$.

\subsection{Organosilanization of silicon and silicon nitride surfaces.}

Silicon oxide is present on the surface of the most common materials used for fabrication of nanomechanical systems such as silicon and silicon nitride. Silicon oxide surface avidly adsorbs water molecules producing silanol groups $(\mathrm{Si}-\mathrm{OH})$ with high density ${ }^{103}$. These groups are used in silanization methods as connection sites that will react with organosilicon molecules forming a silicon-oxygen-silicon carbon linkage. This bonding is thermodynamically and hydrolytically stable. Organosilicon molecules are composed of a small organic group such as methyl, a reactive group such as chloride, methoxy and ethoxy and a terminal group that can be replaced with different functional groups like amino, carboxyl or thiol groups that provides the desired properties for the modified surface ${ }^{104}$. The simplicity of the reaction and the high stability of the product make this method a customary surface modification route. The silanization reaction is usually performed dipping the surfaces into a solution containing the organosilicon molecule, therefore, both sides get modified if the cantilevers are functionalized using this approach. Therefore this method is more suitable for biosensors 
based on nanomechanical resonators ${ }^{37,105}$ that do not require the functionalization asymmetry needed in nanomechanical biosensors based on the surface stress.

\subsection{Immobilization of receptors in arrays.}

Arrays of tens and hundreds of identical nanomechanical systems can be routinely fabricated with current semiconductor technology ${ }^{106}$. This attribute is relevant in biomedical applications such as biomarker discovery and disease detection in which detection from a few to even hundreds of molecules is required. Thus high-throughput methods for reliable functionalization of individual nanomechanical systems in dense arrays are increasingly demanding. In addition, these methods are required to obtain nanomechanical elements that serve as internal reference to reject common noise and drift signals from variations in temperature, relative humidity, pressure, ionic strength and $\mathrm{pH}$ in experiments in liquids, hydrodynamic forces, etc ${ }^{46-47}$. These side effects often mask the sought specific binding events. Reference nanomechanical elements can be obtained by passivating them with molecules that exhibit ultralow fouling. Currently, three methods have been demonstrated for the functionalization of individual nanomechanical elements in microcantilever arrays: incubation into arrays of glass microcapillaries, inkjet delivery and microcontact printing.

In the first method, all the microcantilevers within the array are inserted into an array of disposable glass microcapillaries filled with the liquid containing the probe molecules. Alternatively, the microcapillary array can be substituted by a microarray of small volume reaction wells. This method has been applied for the functionalization of cantilever arrays with different thiolated DNA probes, viral receptors and proteins ${ }^{46-47,51,107}$. The main limitation of this method is that the cantilevers must usually be spaced within the array at least $250 \mu \mathrm{m}$ (the microcapillary diameter). In addition, alignment and handling of the microcapillaries is technically difficult. Inkjet spotting allows delivery of picoliters at the wished positions with an accuracy of $10 \mu \mathrm{m}$. By adjusting the number and volume of microdroplets spotted on the cantilever, a homogeneous and reproducible film can be obtained within seconds. Ink-jet coating has been demonstrated in microcantilever arrays for functionalization with DNA probes $^{108}$ and for positioning bacterial cells on microcantilevers ${ }^{80,109}$. Recently, a method based on microcontact printing has been successfully developed for biofunctionalization of a large-scale array of nanocantilevers ${ }^{110}$. The method relies on the delivery of the molecules from the grooves of the stamp while its base sits on the chip, thus providing the needed mechanical stability to avoid breaking the cantilevers. 


\section{EXAMPLES OF NANOMECHANICAL BIOSENSOR DEVICES AND APPLICATIONS.}

Here, we describe some relevant examples of biosensor devices based on nanomechanical systems that harness some of the mechanical effects described in section 2 . This section does not intend to exhaustively list the intense activity in nanomechanical biosensing. For a more exhaustive revision of applications, we refer the reader to several excellent reviews ${ }^{14-17,20,34-35}$. The aim is to provide a flavor on the different nanomechanical approaches that can be adopted for measuring biological interactions and properties. The most suitable approach depends on a number of factors such as biomolecule size, biomolecule properties, need of label-free analysis, required reliability, needed detection limits, required specificity, required throughput, whether the detection should be ex-situ or in-situ, whether the application is a bioassay based on binding affinity or aims to classify biomolecules in terms of mass and/or nanomechanical properties, etc.

\subsection{Surface stress biosensors}

A surface stress biosensor measures the bending of a nanomechanical system, mostly a cantilever, caused by changes in the surface stress induced by the binding of the targeted molecule to the biomolecular receptors anchored on one side of the cantilever (active side). The opposite cantilever side (passive side) must be inert to the target. Ideally, the passive surface must be blocked to avoid non-specific adsorption. The most sensitive and significant reported bioassays with nanomechanical systems are based on the surface stress mechanism ${ }^{14-20,46-54}$. The surface stress induced bending mechanism is described in section 2.1.1. Biomolecular recognition generates small amounts of surface stress $\left(0.001-0.01 \mathrm{Nm}^{-1}\right)$ which is equivalent to cantilever deflections from few nanometers to tens of nanometers that can be easily measured by optical and electrical methods (see section 1.3.1.). When the surface stress is negative, it is referred to as compressive and induces the elastic surface expansion. Contrarily, when the surface stress is positive, it is referred to as tensile and gives rise to the surface contraction. In most of the significant biological measurements with these techniques ${ }^{46-47,111}$ : i) microcantilever arrays are placed in a fluid cell with inlet and outlet channels for external tubing with the buffer and analyte solutions, ii) the free ends of the cantilevers are sequentially detected by the optical beam deflection method, iii) the displacement is monitored in real-time to detect the changes that the cantilever undergoes when the sample solution flows over the cantilever, iv) the cantilevers within the array are differentially functionalized by microcapillary- or inkjet- based techniques (see sect. 3.3.), v) the temperature is accurately controlled and kept constant, and vi) the differential deflection 
of the cantilevers with respect to reference cantilevers is analysed to reject common sources of drift and noise (see sect. 3.3.).

The label-free capability of surface stress biosensors is remarkable. The most extended bioassay techniques in the biomedical field such as microarrays, radiolabelling-based assays and enzyme-linked immunosorbent assays (ELISA), require labeling of the targeted molecules with fluorescent dyes, radioisotopes, or colorimetric enzymes to be subsequently detected by well-established optical and radioactive readout techniques. The labeling often alters surface characteristics and natural activities of the tagged molecules. Moreover, the labeling procedure is laborious, lengthy and limits the number and types of targets that can be studied.

\subsubsection{Experiments}

The potential of nanomechanical systems for label-free biological sensing was revealed in 2000 when Fritz and co-workers demonstrated that DNA hybridization can be detected in real-time by cantilever sensors. They measured the bending difference between two cantilevers within an array previously functionalized with a complementary and non complementary single stranded (ss) DNA probes, when the targeted ssDNA was flowed over the cantilevers ${ }^{99}$. In these experiments, the authors demonstrated the ability of the technique to detect single-base mismatches. This report inspired later works, in which the methods (cantilever fabrication, immobilization of the ssDNA probes, experimental set-up) were refined to achieve higher sensitivity and to get further insight into the molecular nature of the surface stress signal upon hybridization ${ }^{47}$. The improved sensitivity was exploited to detect mRNA biomarker candidates in total cellular RNA ${ }^{46}$. This study demonstrates the enormous potential of the technique for measuring differential gene expression and for disease biomarker discovery.

Microcantilever surface stress biosensors have also been applied for the detection of protein biomarkers such as prostate-specific antigen (PSA) in a background of human serum albumin (HSA) and human plasminogen (HP) ${ }^{102}$; and creatin kinase and myoglobulin that are acute myocardial infarction biomarkers proteins ${ }^{51}$. The identification and quantification of carbohydrate interactions with other biomolecules is important to understand the biological function of carbohydrates. To this end, Gruber and co-workers described the development of cantilever array biosensors with a carbohydrate-based sensing layer that selectively and sensitively detects carbohydrate-protein binding interactions ${ }^{50}$. Recently, it was reported the first measurement of conformational changes of viral ATPases that shows the potential of 
these nanomechanical tools to study how viruses package their DNA into their capsids ${ }^{53}$. In all the experiments described until now, the biological molecular recognition occurred in situ and in real time, within the fluid. In 2008, Mertens et al harnessed the important role of the hydration forces in highly-packed DNA monolayers to detect ex situ hybridization at the femtomolar level ${ }^{49}$. The experiments were performed in an environmental chamber in which the relative humidity was controlled. It was observed that the surface stress undergoes large changes with the relative humidity, and more importantly, the pattern of these changes depends on the DNA conformation: single stranded or double stranded.

In the field of pathogen detection and treatment, surface stress biosensors have reported significant results. For instance, these devices have shed light into the mechanisms of antibiotic interactions with mucopeptides (components of bacterial cell wall) down to a sensitivity of $10 \mathrm{nM}$, and at clinically relevant concentrations in blood serum ${ }^{111}$. These studies can offer new clues on the mechanisms that make bacteria strains increasingly resistant to antibiotics. Recently, cantilever arrays functionalized with different carbohydrate layers have been applied for real-time discrimination of several Escherichia coli strains in solution ${ }^{48}$.

\subsubsection{Mechanism of surface stress generation upon molecular recognition.}

Surface stress is the amount of reversible work per unit area needed to elastically stretch a pre-existing surface. There are two mechanisms for generation of surface stress upon molecular adsorption ${ }^{43-45}$. The first mechanism arises from the redistribution of the electron cloud surrounding the surface atoms that modifies the inter-atomic interactions. This source of surface stress is significant in chemisorptions processes where electron transfer between the adsorbate and the surface atoms may occur. The surface stress induced by this mechanism is linearly proportional to the surface coverage. This mechanism is the origin of large surface stress variations on gold-coated microcantilevers during self-assembly of alkanethiols or biomolecules (DNA, proteins) derivatized with a thiol group ${ }^{43,100}$. However, this contribution is, in general, negligible during biomolecular recognition processes as the ligand is separated from the surface several nanometers (the height of the receptor). The second mechanism comes from the in-plane interactions that emerge between the biomolecular complexes on the surface such as electrostatic forces, hydrogen bonding, the force induced by the entropy cost of restricting molecular motions, and the desolvation interactions such as the burial of hydrophobic surface area and the consequent release of hydration water layers to the bulk solvent during molecular recognition ${ }^{43,79,112-113}$. The surface stress induced by this mechanism 
exhibits a non-linear dependence on the surface coverage, and it steeply increases when the coverage is near saturation ${ }^{44}$.

In general, nanomechanical biosensors based on surface stress require high packing densities of biomolecular receptors to maximize the response to the targeted ligand. In other words, the generation of significant amount of surface stress requires highly crowded surfaces in order to maximize the repulsive physical steric interactions, ion osmotic pressure and hydration forces. This feature of surface stress biosensors is unique with regards to biosensors in general, in which the density of receptors on the surface is adjusted in order to decrease the steric hindrance and enhance the target binding efficiency ${ }^{47,100}$. In this sense, Ndyiera et $a l$ developed a very interesting model referred to as nanomechanical percolation (figure 12). They noted that, in general, molecular recognition on the cantilever surface is not able to generate a detectable surface stress signal below a critical surface density of receptors. This means that surface stress generation is a collective phenomenon that requires a critical coverage of the surface to achieve connectivity between all the functionalized regions of the surface. The degree of percolation and the strength of the interactions between the surfacegrafted receptors determine the amount of surface stress.

Modeling of the response of surface stress biosensors is very challenging due to the large span of scales that govern the cantilever response, from the molecular recognition interaction, to the long-range molecular interactions to the mechanical response of a mesoscopic element. Sushko et al developed a multiscale model in which atomistic simulations revealed that the biaxial elastic modulus of the biological layer plays a key role in the cantilever response as pointed in section 2.3.1. 


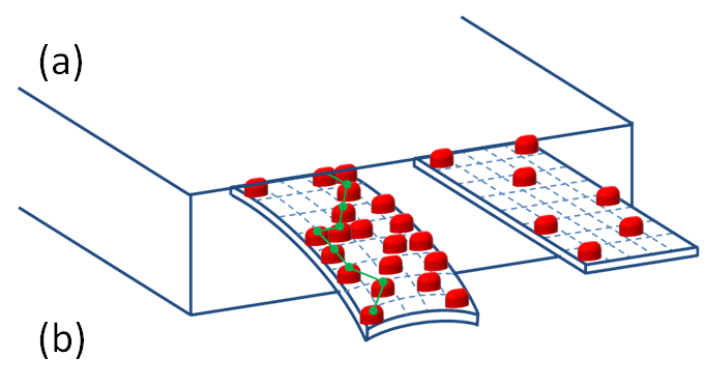

(b)

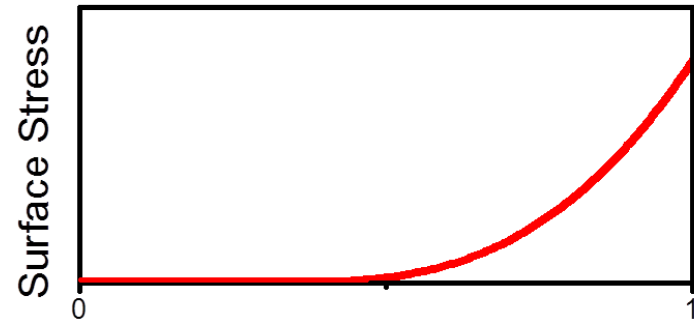

Coverage

Figure 12. (a) Schematics of the concept of the nanomechanical percolation model. The cantilever bending induced by surface stress requires that the adsorbate coverage is above a threshold to establish interaction-connectivity over the microcantilever surface. (b) A surface stress curve derived from the percolation model.

\subsection{Biosensors based on nanomechanical resonators}

Nanomechanical resonators as shown in section 2.2. have demonstrated unprecedented detection limits in mass sensing of atoms and molecules in vacuum. The mass detection limits have been recently pushed down to the yoctogram range, i.e., the mass of a single proton can be measured. Two ingredients are essential to achieve high mass sensitivity: devices with nanoscale dimensions and high quality factors (1000-100 000) that implies measurements in vacuum. However, sensing of biomolecules should ideally be carried out in aqueous solutions, the natural environment where biological processes occur. Nanomechanical resonators in liquids exhibit a very low quality factor (1-10) as a consequence of the viscous damping. In addition, the liquid is dragged along with the nanomechanical resonator, increases its effective mass and thus lowers the sensitivity. The miniaturization of the devices to the nanoscale does not improve these limitations ${ }^{91-92,114}$. More importantly, biological detection requires many repetitive measurements that can only be achieved with disposable and cost-effective devices that can be easily both handled and measured. These requirements are fulfilled by microcantilever arrays that are commercially available, but not by state of the art nanoscale mechanical resonators that are still fabricated at low rate by nanofabrication techniques and are highly irreproducible in the dimensions and mechanical response. In addition, the measurement of the resonant frequency of these devices in liquid is scientifically and technically challenging. These limitations have limited the success of nanomechanical resonators as real-time biological sensors, as can be inferred from the achievements of nanomechanical resonators in the biological context shown in figure 13 in comparison with the achievements in mass sensing (figure 8). 


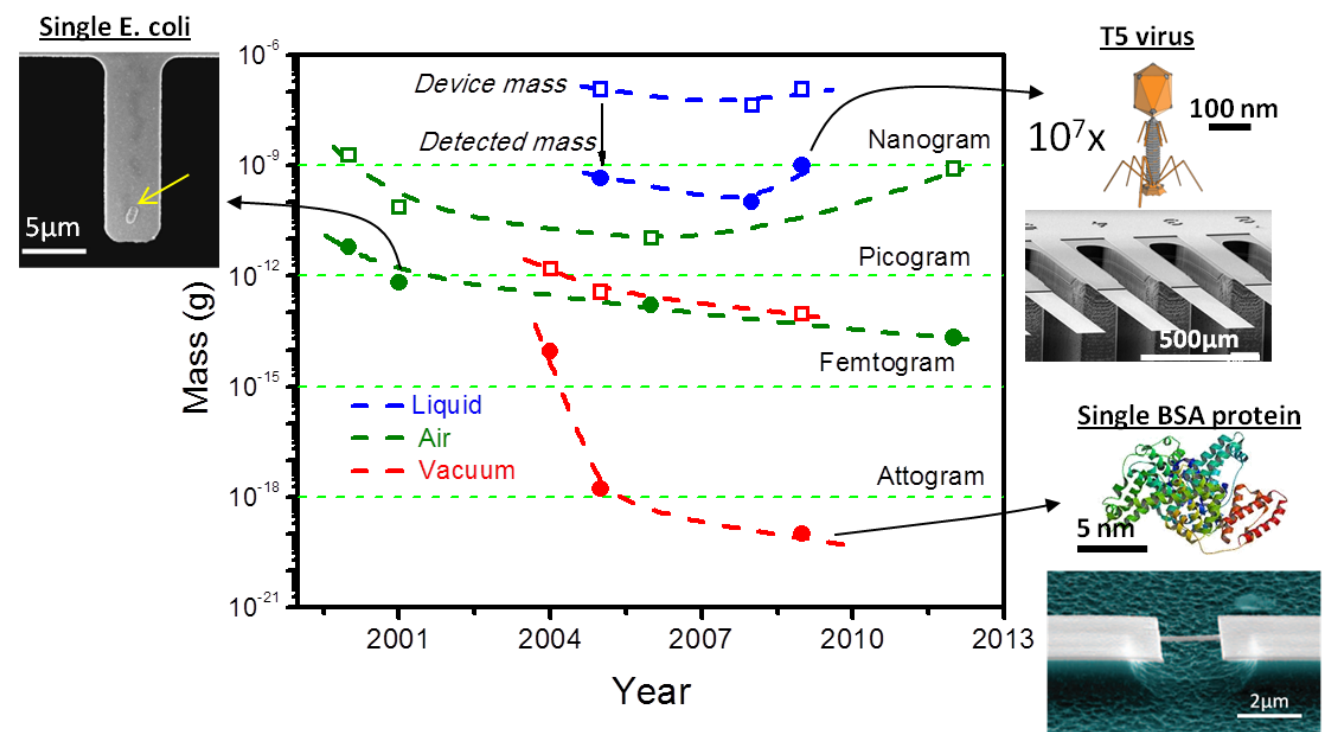

Figure 13. Chronology of the achievements in label-free biological detection based on nanomechanical resonators. The device mass and the detected mass levels are plotted as empty and filled symbols, respectively. The data split into measurements in vacuum ${ }^{115-117}$ (red), air ${ }^{94,118-120}$ (green) and liquid ${ }^{107,121-122}$ (blue). In air and vacuum, the measurements are usually performed ex-situ before and after the biological assay to attain the highest sensitivity in the resonance frequency due to the high quality factor. However, non-specific adsorption and contamination during desiccation can lead to errors. The last data point is obtained from recent work in a nanoelectromechanical-mass spectrometry system, in which proteins are introduced by electrospray injection from the fluid phase in ambient conditions into vacuum, and are subsequently delivered to a ultrahigh-frequency nanoelectromechanical resonator by hexapole ion optics ${ }^{117}$. Precipitous frequency shifts are recorded in real time as analytes adsorb, one by one, onto the resonator. Measurements in liquid are able to monitor in real-time the biomolecular binding events. However, the mass sensitivity is severely degraded due to the low quality factor. Due to the scarce data in biological assays, the first point is obtained from the adsorption of biotinylated latex beads (diameter $250 \mathrm{~nm}$ ) on streptavidin-activated cantilevers ${ }^{121}$, and the second point is derived from measurements in real-time of the change of the fluid properties when glycerol is added to water ${ }^{122}$. In the first ${ }^{121}$ and third ${ }^{107}$ data points in liquid, the quality factor is enhanced by using high vibrational modes, whereas for the second point self-excitation of a selected vibration mode of a microcantilever in liquid is used, achieving a frequency stability of the order of $1 \mathrm{ppm}$ for a bandwidth of $1 \mathrm{~Hz}^{122}$. The arrows point out to the devices and detected biological systems: a single $E$. coli weighed in air (at the left) ${ }^{119}$, the binding of bacterial viruses to cantilevers activated with bacterial protein receptors in liquid (at the top right corner) ${ }^{107}$, a single BSA protein delivered in vacuum by mass spectroscopy technology to a high frequency nanoelectromechanical resonator (at the bottom right corner) ${ }^{117}$.

There are three strategies addressed to exploit the unprecedented attributes of nanomechanical resonators for biological detection: i) measurements in liquid with the development of excitation techniques addressed to enhance the quality factor, ii) ex-situ measurements in air and vacuum before and after the biological assay, iii) transport and delivery of biomolecules in vacuum to the nanomechanical resonator, and iv) innovative designs of nanomechanical resonators. We summarize the findings obtained with these different approaches.

\subsubsection{Measurements in liquids.}

Ghatkeshar, Braun et al proposed to enhance the quality factor of microcantilevers in liquid by exciting their higher vibration modes ${ }^{121,123}$. They observed that the quality factor increased from 1 at first mode to 30 at $16^{\text {th }}$ mode. The apparent mass of the liquid displaced by 
the oscillating cantilevers decreased asymptotically with mode number. Thus the used cantilever has to displace almost 40 times its own mass at fundamental vibration mode and about 10 times at $16^{\text {th }}$ mode. In these experiments, the microcantilever array is directly mounted onto a piezoelectric actuator in order to obtain a perfect mechanical matching. The technical difficulty lies on keeping the piezoelectric actuator and electrical contacts insulated. This method has been applied to detect bacteriophage virus T5 interactions with a membrane protein receptor-the FhuA receptor of Escherichia coli- at subpicomolar concentrations ${ }^{107}$. In this work, experiments were performed in a microarray format, allowing differential measurements of the sensing (FhuA-functionalized and casein-blocked) and reference (casein functionalized only) cantilevers, in parallel, under the same physiological conditions. This feature allows the sensitivity of the measurements to be improved when temperature drifts or non-specific binding occurs.

Alternatively, Ramos et al have implemented photothermal actuation for the selfexcitation of a selected vibration mode of a microcantilever in liquid ${ }^{122,124}$. The gain of the positive feedback loop is adjusted in order to obtain a negative effective damping. In this regime, the amplitude noise is squeezed due to the nonlinear saturation of the system and the phase noise is largely reduced. In this way, the microcantilever vibration achieves frequency stability of the order of $1 \mathrm{ppm}$ for a bandwidth of $1 \mathrm{~Hz}$.

\subsubsection{Ex-situ measurements in air and vacuum.}

An alternative approach to circumvent the low $Q$ problem has been the dip-rinsemeasure approach. Ramos et al. used this approach to follow the hybridization of complementary DNA strands on defined regions of a nanocantilever ${ }^{101}$. Reaction sites were predefined by gold patterning of small areas either at the clamping or at the nanocantilever free end (see below section 4.2.5 and fig. 16(a)). Such confinement allowed disentangling the signal arising from stiffness effects in the biolayer from the added mass effect. In this experiment, the follow-up of rigidity effects near the clamp demonstrated superior performance to the detection based on the mass effect.

The main limitation of the dip and rinse approach is that unspecific binding and contamination during desiccation degrade the otherwise very low detection limits, so careful blocking of the non-binding sites of the resonator surface and rising of the devices are critical. Still, Craighead and co-workers have successfully used this method together with secondary 
mass labeling with nanoparticles to achieve impressive detection of $50 \mathrm{fg} / \mathrm{mL}$ of the prostate specific antigen in very demanding samples such as serum ${ }^{37}$.

\subsubsection{Nanomechanical mass spectrometry.}

Mass spectrometry (MS) for biological applications concerns the identification of biomolecules according to their mass-to-charge ratios ${ }^{125}$. Thus, conventional mass spectrometers require the ionization of the analyte molecules, which implies limitations in terms of sensitivity (that decreases for increasing mass-to-charge ratios), and suitability (some molecules are difficult or too fragile to ionize). Roukes' group has recently applied nanomechanical resonators for biomolecular spectrometry. In their first demonstration, nanoparticles and protein species are introduced by electrospray injection from the fluid phase in ambient conditions into vacuum, and are subsequently delivered to a high frequency nanoelectroemechanical resonator (figure 14). Remarkably, jumps in the resonance frequency are recorded in real time as analytes adsorb, one by one, onto the resonator. The problem is that the frequency shift, even if the stiffness effect is neglected, depends on the adsorption position in addition to the mass (see section 2.2.1.). The authors have recently refined the technique for simultaneously measuring the resonance frequency of the first two vibration modes. Discrete, time-correlated frequency jumps of these modes enabled the real-time mass measurement of IgM protein, one by one.

Conventional mass spectrometers are not capable of attaining high mass resolution in complex protein assemblies which is key to obtain a better understanding of the role of molecular associations in the cell processes. More importantly, the sample ionization and fragmentation into small peptides result into a complex analytical process whose significance may be far from the native biological conformation ${ }^{126}$. Nanomechanical resonators offer significant promise because they do not require high ionization that can induce structural changes in the proteins, and have potential to resolve minute mass variations in "intact" large protein complexes $\left(\sim 10^{5} \mathrm{Da}\right)$. In addition, recent experiments that exploit the two dimensional vibration features of 1D nanobeams, such as silicon nanowires and carbon nanotubes, show promise for biological nanomechanical spectroscopy by classifying macromolecules according to their mass with zeptogram sensitivity and their Young's modulus with a sensitivity to variations of 100 parts per million (see below section 4.2 .5 and figure $16(d))^{127}$. 

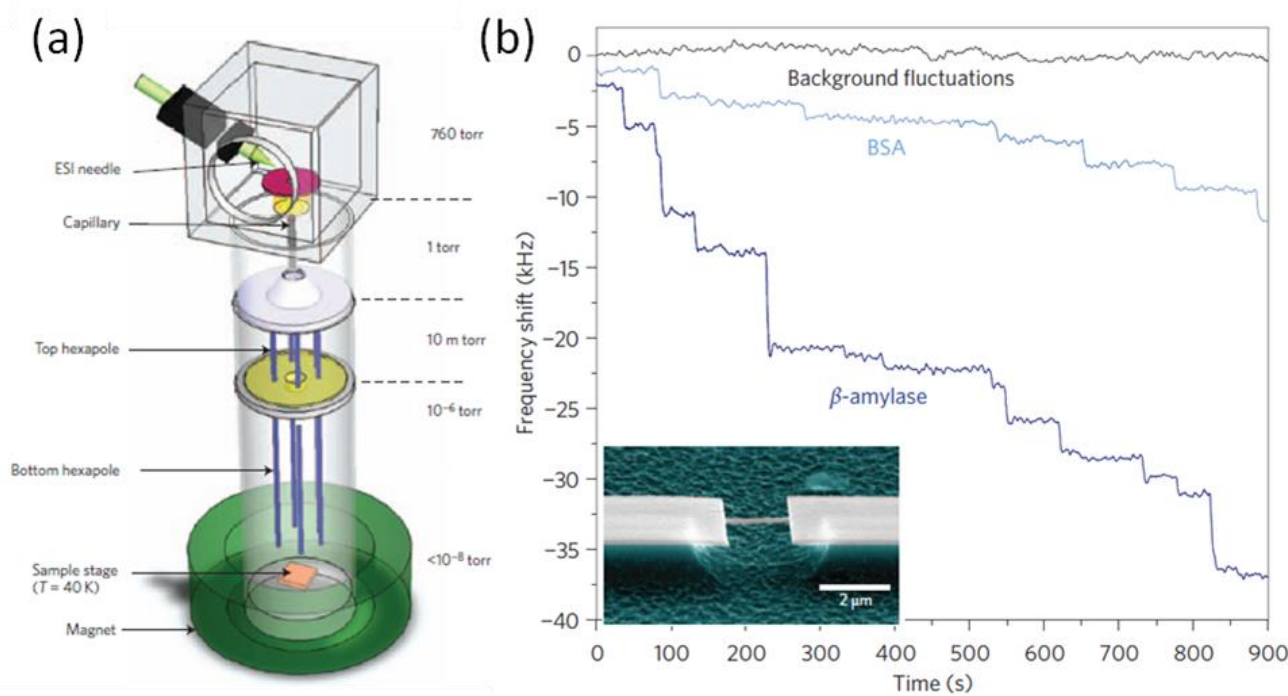

Figure 14. (a) Schematic depiction of the nanomechanical protein spectrometer developed by Roukes' group in reference ${ }^{117}$. Protein ions are produced and stripped of fluidic solvent in the course of fluid-phase electrospray ionization (ESI). These bare ions traverse through a three-stage differentially pumped vacuum system and land onto the nanomechanical resonator. Two stages of hexapole ion optics driven at radio frequency are used to guide the species to the nanomechanical resonator. (b) As the individual protein molecules accrete onto the resonator one by one, downward resonant frequency jumps are recorded. The experimental data show the distinctly different precipitous resonance frequency shifts during ESI-induced adsorption of BSA (66 kDa) and b-amylase (200 kDa). In the inset, a scanning electron microscopy image of the doubly clamped beam devices used in these experiments. Devices are embedded in a nanofabricated three-terminal UHF bridge circuit for electrical readout. Adapted reproduction with permission of Naik et $a^{117}$.

\subsubsection{Innovative nanomechanical resonators.}

Resonant microchannels. Manalis' group designed cantilevers with inside channels for the liquid flow, so these suspended microchannel resonators (SMRs) can thus be operated in vacuum at very high quality factors of up to $150000^{128}$. Later, they applied the devices for biological measurements ${ }^{129}$. Thus, the inside walls of the microchannels were functionalized with antibodies to measure the kinetics of antibody-antigen reactions with a level of sensitivity of $100 \mathrm{ng} / \mathrm{mL}$. The only label-free biosensor that can achieve a similar detection limit is the quartz microbalance, but for the case of the SMRs the amount of sample needed is minimal as the microchannels may contain volumes as low as $10 \mathrm{pL}$. In the same work, the SMRs were applied to track passing particles of diverse sizes, such as bacteria or cells. When the particle travels the microchannel, the resonant frequency drops by an amount that depends on the position of the particle along the resonating structure and on the particle density and volume (or equivalently, the particle buoyant mass). The bacteria Escherichia coli and Bacilus subtilis, which have different masses, were distinguished with SMRs. Recently, this group measured the buoyant mass of cells in two fluids of different densities in order to determine single-cell mass, volume, and density (figure 15(a)). It was found that the cell density was an excellent fingerprint of cellular processes that would be otherwise undetectable by mass or volume 
measurements. This key concept was demonstrated in four studies: identifying Plasmodium falciparum malaria-infected erythrocytes in a culture, distinguishing transfused blood cells from a patient's own blood, identifying irreversibly sickled cells in a sickle cell patient, and identifying leukemia cells in the early stages of response to a drug treatment ${ }^{130}$.

(a)

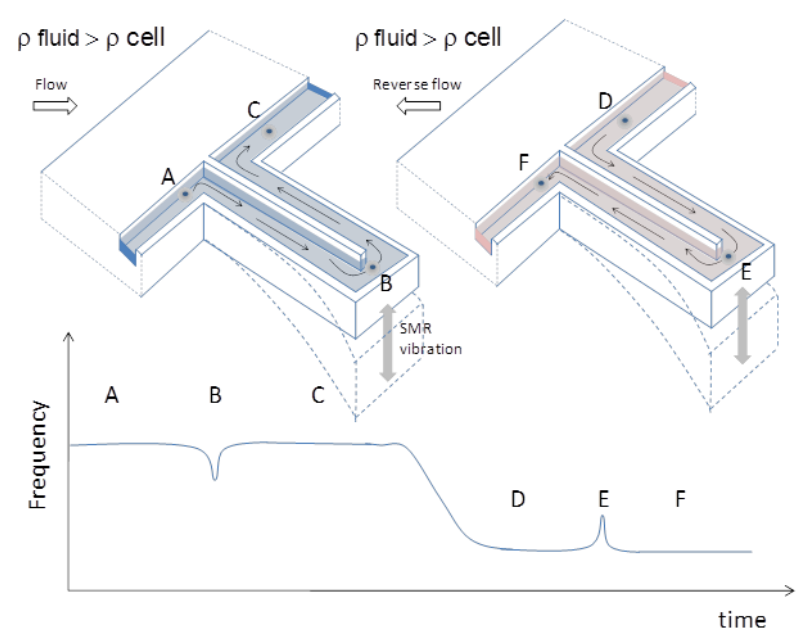

(b)

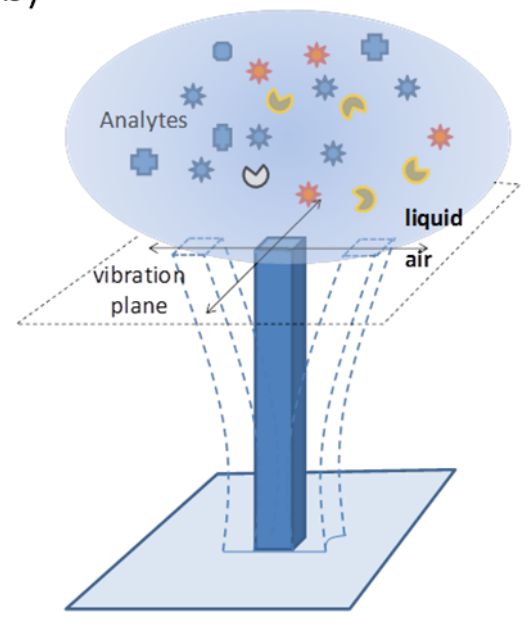

Figure 15. (a) Measurement of the mass and density of cells with suspended microchannel resonators. Measurement starts with microchannel filled with a media less dense than the cell (blue fluid position A). The density of the fluid is determined from the baseline resonance frequency. When the cell travels through position $B$ the buoyant mass of the cell in the blue fluid is determined from the resonance shift. A second fluid of higher density than the cell is passed through the channel by reversing the flow direction. The resonance shifts to lower values due to the increase in the density of the fluid filling the channel. The buoyant mass of the same cell is then measured again as the cell travel the resonator apex (position E) but now in the red fluid. The measurements of fluid density and cell buoyant masses in red and blue fluid provide the mass, volume and density of the passing cell. See references ${ }^{129-130}$. (b) Schematics of the resonance of a micropillar when only the top surface is wetted by the liquid. The vibration plane is parallel to the liquid/air interface and the beam is resonating in air thus the quality factor is not degraded by viscous damping and drag of the fluid on the largest section of the resonator. See references $^{131-132}$

Micropillar resonators. Contrarily to the widespread fabrication of the nanomechanical resonators parallel to substrate or chip, micropillars are vertically oriented ${ }^{131-132}$. Tailoring the arrangement of micropillar arrays the surface can be transformed in superhydrophobic with large contact angles of up to 150 degrees. Thus, a deposited droplet of biological solutions contacts just the top side of the micropillars (figure 15 (b)). Since the vibration plane is parallel to the liquid-air interface, as it occurs with quartz crystal microbalance, the micropillar exhibits high quality factors of 500 in liquid. DNA hybridization has been followed in real-time with micropillar resonators without the need of labels at $10 \mathrm{nM}$ target concentration ${ }^{132}$. 


\subsubsection{Uncoupling mass and stiffness.}

As described in sections 2.1.2, 2.2.1. and 2.3.2., the adsorbate-induced shift of the resonance frequency of a nanomechanical resonator arises from three effects: added mass (negative shift), mechanical stiffness (positive shift), and surface stress (either positive or negative shift). The last effect is usually the smallest of the three, and it can be neglected. However, it can be significantly enhanced by designing nanomechanical resonators with geometries and dimensions that amplify the non-linear effects of surface stress ${ }^{67}$. The stiffness effect has received little attention, despite its importance and potential. Experiments and theory unequivocally teach us that the resonance frequency is a convolution of the stiffness and mass of the adsorbate. Since both effects exhibit opposite signs, the sensitivity can be reduced and the mass underestimated. There are four methods that turn this apparent disadvantage into a striking unique asset, enabling the simultaneous measurement of the mass and stiffness induced by the biomolecular interaction on the resonator surface. These methods are summarized below.

- Arrays of dual nanomechanical resonators. The arrays consist of small and thin cantilevers (thickness below $100 \mathrm{~nm}$ ) with sensing gold areas alternately placed on the free and fixed cantilever ends (fig. 16(a)). The Au areas act as sensing regions as they can be functionalized by means of thiol chemistry. The nanomechanical arrays provide a double flavor of the adsorbed molecules: the added mass reported by the cantilevers with the $\mathrm{Au}$ area at the tip and the nanoscale elasticity reported by the cantilevers with the Au area at the clamp ${ }^{101}$. See section 4.2.2. for more experimental details and section 2.3.2. for the nanomechanical basis of these devices.

- Measuring the quality factor. In dissipative media such as air and liquids, the quality factor provides an extra channel to quantify adsorption. The adsorbate mass and stiffness can be accurately determined by combining the measurements of the resonance frequency and quality factor of nanomechanical resonators (fig. 16(b) $)^{78}$. See section 2.2.2.

- Electrostatic pull in instability method. When a voltage is applied between a cantilever and the substrate, the electrostatic force deflects the cantilever. When the gradient of this force overcomes the spring constant of the cantilevers, the cantilever jumps to contact the substrate surface due to the electromechanical instability (fig. 16(c)). Thus, cantilever spring constant and its modification by the mechanical properties of the adsorbates can be obtained by measuring the voltage value at which the electrostatic pull in instability occurs $^{133-134}$. 
- Two dimensional vibrations of 1D nanobeams. Whereas a resonator fabricated by topdown methods can vibrate in just one dimension because it is shaped like a diving board, a perfectly axisymmetric one-dimensional nanoresonator such as a nanowire or nanotube can support vibrations with the same amplitude and frequency in two dimensions. A molecule landing on such a nanoresonator will break this symmetry and the vibration will become a superposition of two orthogonal vibrations with different frequencies (fig. 16(d)). The measurement of the frequency degeneration breakage enables the determination of the adsorbate's mass and stiffness ${ }^{127}$.

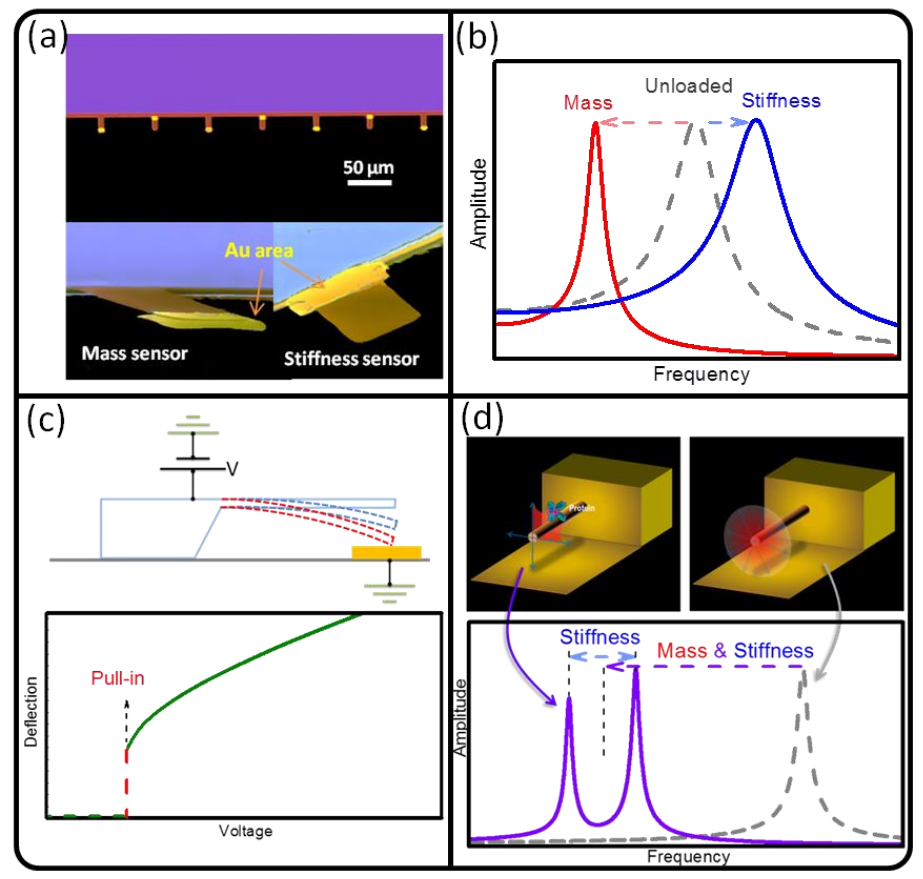

Figure 16. Schematics of the methods for disentangling mass and stiffness effects on the response of nanomechanical resonators. (a) Arrays of dual nanomechanical resonators ${ }^{101}$. Top: Optical image of the array of nanomechanical sensors. Left- and right bottom corners: scanning electron microscopy images of a cantilever with the Au area at the free end (mass sensor) and a cantilever with the Au area at the fixed end (stiffness sensor), respectively. The cantilevers are $15 \mu \mathrm{m}$ long, $6 \mu \mathrm{m}$ wide, $100 \mathrm{~nm}$ thick, and with a cantilever pitch of $60 \mu \mathrm{m}$. (b). Measuring the quality factor ${ }^{78}$. Effect of stiffness and mass on the resonance frequency peak (exaggerated hypothetical case for the sake of clarity). The stiffness induces a positive shift of the resonance frequency, and a negligible change of the quality factor. On the contrary, the mass induces a negative shift of the resonance frequency and an increase of the quality factor. By measuring both parameters, the stiffness and mass of the adsorbate can be uncoupled. (c) Electrostatic pull in instability method ${ }^{133-134}$. A voltage is applied between a cantilever and an electrode on the substrate underneath. The electrostatic force deflects the cantilever. There is a threshold voltage for which cantilever abruptly snaps into the electrode when the force gradient equals the spring constant of the cantilever. (d) Two dimensional vibrations of 1D nanobeams ${ }^{127}$. Generic resonance curves of 1D nanobeams (nanowires, nanotubes) and schematic depictions of the vibrations. If the nanobeam is perfectly axisymmetric, it vibrates in all the transverse directions with the same resonance frequency. When a protein lands on a such a nanoresonator, the frequency degeneration is broken, and the fundamental vibration mode splits into two orthogonal vibrations with different frequencies. The mean shift of the two resonance frequencies depends on the mass and the stiffness of the adsorbate. In turn, the frequency difference only depends on the adsorbate stiffness. Thus, stiffness and mass effects can be decoupled by measuring the frequency degeneration breakage. 


\section{DETECTION LIMITS.}

\subsection{The bottom line: ultimate detection limits}

Systems that dissipate energy necessarily are sources of noise; the converse is also often true. This is the basic statement of the fluctuation-dissipation theorem ${ }^{135}$. For instance, a microcantilever in fluid fluctuates with respect to the rest position mainly due to the random impacts of the surrounding molecules. In the same way, the cantilever dissipates the stored mechanical energy through its interaction with the surrounding molecules that constitute in this case the thermal bath. Thermomechanical noise determines the ultimate detection limits of nanomechanical biosensors in their different operation modes: static ${ }^{136}$ and dynamic ${ }^{135,137}$. The fluctuation of the mesoscopic nanomechanical system can be modeled as the effect of a force that represents the fast atomistic collisions that establish the thermal bath. The thermal force $\left(F_{t h}\right)$ is a force uncorrelated in time and space that exhibits a Gaussian distribution around zero. The white spectral density of the thermal force for singly and doubly clamped beams for each vibration mode $n$ is given by ${ }^{135,138}$,

$S_{t h, n} \approx \frac{4 k_{B} T m_{b} f_{n}}{Q}$

where $k_{B}$ is the Boltzmann's constant and $T$ is the temperature. The amplitude noise spectral density is given by the dynamic susceptibility times $S_{t h, n}{ }^{135,138}$,

$S_{w, n}(\omega) \approx \frac{4 \pi k_{B} T f_{n}}{m_{b} Q\left[\left(f^{2}-f_{n}{ }^{2}\right)^{2}+\frac{f^{2} f_{n}^{2}}{Q^{2}}\right]}$

The average displacement fluctuation $\left\langle w_{n}{ }^{2}\right\rangle$ is then given by integrating equation (13) from $f=0$ to infinity, which gives the well-known equipartition theorem result $\left\langle w_{n}{ }^{2}\right\rangle=\frac{k_{B} T}{k_{n}}$ where $k_{n}$ is the effective spring constant given by $k_{n}=4 \pi^{2} m_{b} f_{n}{ }^{2}$. The static noise is calculated by integrating in the low frequency range which gives,

$\left\langle w_{n}{ }^{2}\right\rangle \approx \frac{1}{2 \pi^{3}} \frac{\mathrm{B} k_{B} T}{m_{b} f_{0}{ }^{3} Q}$

where $B$ is the frequency bandwidth of the measurement (inversely proportional to the measurement acquisition time). The minimal variation of surface stress that can be detected using a microcantilever and measuring the the displacement of the free end is given by, 
$\Delta \sigma_{\min } \approx \frac{E h^{2}}{3 L^{2}(1-v)} \sqrt{\frac{1}{2 \pi^{3}} \frac{\mathrm{B} k_{B} T}{m_{b} f_{0}{ }^{3} Q}}$

Following a similar reasoning, for a cantilever oscillating at one of its natural frequencies $f_{n}$, the mean-square frequency due to thermal noise is given by ${ }^{139}$

$\left\langle\Delta f_{t h, n}{ }^{2}\right\rangle \approx \frac{B k_{B} T}{4 A^{2} \pi^{2} m_{b} Q f_{n}}$

where $A$ is the oscillation amplitude. The minimal mass and minimal stiffness that can be detected by confining the adsorption to the free and fixed ends of a microcantilever is respectively given by (see section 2.3.2 and eq. (10)),

$\Delta m_{\min } \approx \sqrt{\frac{m_{b} B k_{B} T}{16 \pi^{2} A^{2} Q f_{n}{ }^{3}}}$

$\left(E_{a} V_{a}\right)_{\min } \approx E_{b} V_{b} \sqrt{\frac{B k_{B} T}{144 \pi^{2} A^{2} m_{b} Q f_{n}{ }^{3}}}$

In the case of uniform adsorption over the resonant beam, if we neglect the stiffness effect, the minimal mass per unit area that can be detected is,

$\left(\frac{\Delta m}{S}\right)_{\min } \approx h \rho_{b} \sqrt{\frac{B k_{B} T}{\pi^{2} A^{2} m_{b} Q f_{n}{ }^{3}}}$

In order to obtain a numerical flavour of the detection limits expressed as equations above, we have calculated the minimal mass and minimal Young's modulus for quasi-punctual biological adsorbates on the free and fixed end of a singly clamped silicon beam that is $5 \mu \mathrm{m}$ long, $0.5 \mu \mathrm{m}$ wide and $50 \mathrm{~nm}$ thick. In addition, we have calculated the minimal mass per unit area for the case in which the biological adsorption is uniformly distributed over the beam. The calculations where performed for quality factors from 1 to 10000 . Representative quality factors of the beam in liquid, air and vacuum approximately range 1-10, 50-500, and 1000-10 000 , respectively. The results of these calculations are plotted in figure 17. The resolution for the case of a punctual mass goes from $50000 \mathrm{Da}$ in liquids to $1000 \mathrm{Da}$ in high vacuum. This implies that the mass of single protein can be detected in liquid by the proposed nanocantilever. Protein masses ranges from $10^{4}$ to $10^{6} \mathrm{Da}$. In the graph, we have marked the 
mass of an antibody $(\approx 150 \mathrm{kDa})$ and the cancer biomarker PSA $(\approx 30 \mathrm{kDa})$. In high vacuum, mass variations of $0.1 \%$ in macromolecular complexes of typically $1 \mathrm{MDa}$ can be detected. The measurements of these variations can be related to changes in the biological function, conformation and interaction with partners of these complexes during relevant cellular processes ${ }^{117,126}$. The plotted the Young's modulus resolution in figure 17 corresponds to two cases: a spherical particle of diameter $120 \mathrm{~nm}$ to simulate the HIV virus in liquid, and a protein of volume of $1000 \mathrm{~nm}^{3}$ in vacuum. In this plot, we use two materials for the beam, silicon and SU8 (as in figure 11 and discussion in section 2.3.2) to highlight the importance of the beam material in these measurements. The HIV Young's varies from $900 \mathrm{MPa}$ to $400 \mathrm{MPa}$ during the maturation process ${ }^{33}$. It is believed that the decrease of the stiffness is a viral mechanism to assist the cell invasion and infection process. Our calculations show that variations in $10 \%$ of Young's modulus of a single viral particle can be recorded in liquid with the proposed silicon cantilever. The resolution can be enhanced 40 times if the beam is fabricated in SU-8. Similarly, variations in the Young's modulus of a single protein of $0.02 \%$ and $50 \mathrm{ppm}$ can be detected with the silicon and SU8 beams, respectively. This potential can be harnessed to distinguish subtle protein variations as mutations and conformational changes that are revealed in the mechanical properties.

Finally, we analyse the detection limits for the case of mass uniformly distributed over the beam (bottom graph in figure 17). This is the practical case of binding affinity assays or when the adsorption position cannot be controlled. The mass resolution of the modeled silicon nanocantilever is of about $10 \mathrm{pg} / \mathrm{cm}^{2}$. This value is 100 times better than the noise floor of quartz crystal microbalance biosensors (QCM), and 5 times better than the limit of surface plasmon resonance biosensors (SPR) estimated from the detection limit in refractive index that approximately scales with the mass. The detection limit achieved by the proposed beam is comparable to that of suspended resonant microchannels (SMRs) in vacuum. Moreover, the minimal total mass that can be detected in liquid by the proposed beam for the case of uniform mass is of about $0.25 \mathrm{ag}$, which is clearly superior to the minimal detectable mass in QCM, SPR and SRM, of 1ng, 1pg and 300 ag, respectively. 
It is worth to notice that the simulated beam exhibits a surface stress resolution of the order of $10^{-8} \mathrm{~N} / \mathrm{m}$ in liquids (data not shown in fig. 17). The surface stress method has been applied for label-free gene fishing in total RNA with a sensitivity $10 \mathrm{pM}$ with a noise floor of $10^{-}$ ${ }^{4} \mathrm{~N} / \mathrm{m}^{46}$. The noise value is four orders of magnitude higher than the ultimate detection limit as a consequence of the non-specific adsorption (revised below), temperature fluctuations, subtle differences in the mechanical properties and geometry between the sensing and reference cantilevers, fluctuations in $\mathrm{pH}$ and ionic strength etc. Therefore, enormous improvement of detection limits can still be expected by the optimization of the instrumentation, device miniaturization, microfluidics implementation and refinement of the fabrication procedures. The achievement of a better surface stress resolution would open the door for gene expression analysis in single cells without the need of sample amplification.

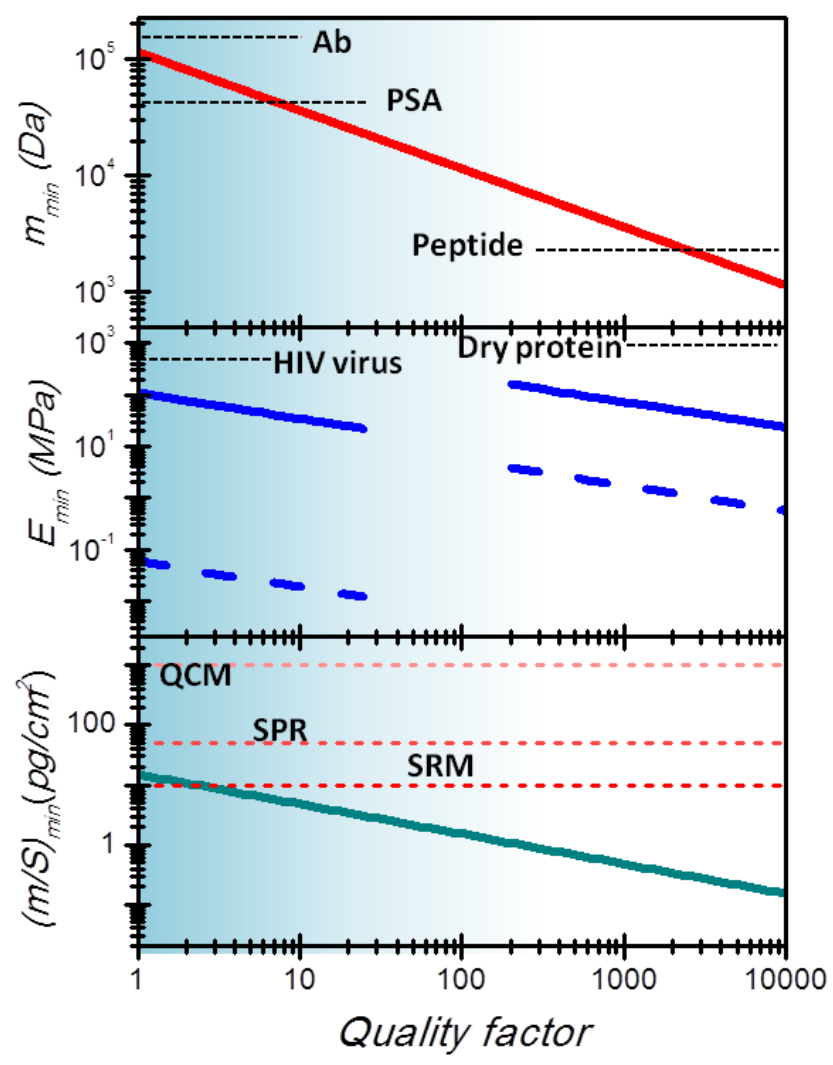

Figure 17. Calculation of the thermomechanical limits in biological detection for a resonant silicon nanocantilever that is $5 \mu \mathrm{m}$ long, $0.5 \mu \mathrm{m}$ wide and $50 \mathrm{~nm}$ thick. Top: Minimal detectable mass for the case of a biomolecule on the free end of a nanocantilever. The mass of an antibody ( $A b)$ and the prostate specific antigen (PSA) and a peptide are marked. Middle: Young's modulus resolution for two cases: a single HIV virus in liquid (Q from 1 to 25 ) and for a single dry protein ( $Q$ from 200 to 10000 ). In both cases, the adsorption site is near the fixed cantilever end. The Young's modulus resolution is plotted for two beam materials, Si (continuous line) and SU8 (dashed line). The approximate values of the Young's modulus of HIV in liquid and dry proteins are also marked. Bottom: Minimal detectable mass per unit area when the mass is uniformly distributed over the nanocantilever. The detection limits of biosensors based on QCM, SPR and SRM are marked for comparison. 


\subsection{Nonspecific adsorption limitation}

The ultimate detection limits predicted above can be far from the real-detection limits when nanomechanical biosensors functionalized with bioreceptors are immersed in complex solutions such as serum to detect the presence of biomarkers in real-time or subsequently exsitu. In this situation, other molecules at much higher concentration, even billion times greater, are present in the solution ${ }^{14}$. Although, these molecules have much lower affinity for the sensor-grafted receptors, their high concentration imposes the real-detection limit. A key feature that a label-free biosensor must fulfill is the selectivity, since the biosensor response can interpret nonspecific adsorption as molecular recognition events (false positive). For instance, cancer biomarkers are in blood plasma at a concentration in the range of $1 \mathrm{ng} / \mathrm{mL}$, whereas the concentration of no-sought proteins is of about $70 \mathrm{mg} / \mathrm{mL}$. The sensitivity to achieve the concentration of cancer biomarkers is complied by most of nanomechanical biosensors. However, the selectivity that determines the rate of false positives and false negatives has received little attention. Detection of cancer biomarkers in complex media such as serum requires of selectivity greater than 1 par per million.

Theoretical predictions indicate that the selectivity required for biomarker detection in complex media can be achieved by functionalizing the sensors with a high receptor surface density ${ }^{140}$. This prediction agrees with the findings in nanomechanical biosensors based on surface stress, in which the best results are obtained at high packing densities of receptors (see section 4.1.2. and references therein). A second theoretical prediction is that additional intermediate surface passivation step by small inert molecules after the receptor-incubation could significantly reduce biofouling and helps in achieving better selectivity. Interestingly, the size and geometry of the blocking molecule used for back-filling of the voids in the sensor surface plays a critical role. This agrees with the results from recent statistical analysis of the effect of immunoreactions on the response of nanomechanical biosensors in the static mode $^{141}$. The study comprised 800 cantilevers with different antibody surface densities, two blocking strategies based polyethylene-glycol (PEG) and bovine serum albumin (BSA), stringent controls with nonspecific antibodies and small proteins such as lysozyme. The study revealed that the performance of the assay critically depends on both antibody surface density and blocking strategies. They found that the optimal conditions involve antibody surface densities near but below saturation and blocking with PEG. 
In addition, other practical approaches have been proposed to minimize the nonspecific adsorption and enhance the selectivity. As pointed out above in section 3.3., the use of arrays of nanomechanical elements with an internal reference helps to reject common noise sources, including nonspecific adsorption. Other approach is the implementation of the sandwich assays traditionally used in ELISA. In this assay, the nanomechanical system is functionalized with a molecular receptor specific to the biomarker of interest. After exposure of the nanomechanical system to the sample, the device is incubated with secondary receptors that tether a molecule that acts as signal amplifier, such as a nanoparticle to increase the mass effect. The use of two different receptors largely enhances the sensitivity and specificity. This approach was applied to detect prion proteins with nanomechanical resonator, which in conformationally altered forms are known to cause neurodegenerative diseases in animals as well as humans ${ }^{37}$. The resonance frequency was detected ex-situ in high vacuum. For the direct incubation of the nanomechanical resonators functionalized with a primary antibody against the prion protein, the detection limit was about $20 \mu \mathrm{g} / \mathrm{mL}$. When the resonators where subjected to a subsequent step of incubation with secondary antibodies tethering, the detection limit was enhanced 3 orders of magnitude, being about $2 \mathrm{ng} / \mathrm{mL}$. The cons of sandwich assays are the major complexity, the availability of two suitable receptors with high binding efficiency and the cross-reactivities in multiplexed assays.

A second promising strategy that keeps the natural label-free feature of nanomechanical biosensors is to implement microfluidics for sample purification and preconcentration. The potential of this approach has been demonstrated with label-free nanowire nanosensors. In this work, a microfluidic purification chip simultaneously captures multiple biomarkers from blood samples and releases them, after washing, into purified buffer for sensing by the nanosensors ${ }^{8}$. This two-stage approach isolates the detector from the complex environment of whole blood, and reduces its minimum required sensitivity by effectively pre-concentrating the biomarkers. The authors demonstrated quantitative and specific detection of two model cancer antigens from a $10 \mathrm{ml}$ sample of whole blood in less than $20 \mathrm{~min}$.

\section{SUMMARY AND OUTLOOK}

Many fundamental biological processes, such as cellular signalling, biomolecular interactions, biomolecular machinery, and disease manifest as or arise from mechanical responses such as piconewton scale forces, subnanometer-scale displacements, elasticity changes, mass variations. These changes easily fall in the range of the detection capabilities of nanomechanical systems that exploit their increasingly small size and high mechanical 
responsivity, i.e., the ability to displace in response to minuscule forces. This review provides insight into the mechanical phenomena that occur in suspended mechanical structures when either biological adsorption or interactions take place on their surface. The phenomena include variations in mass, surface stress, effective Young's modulus and viscoelasticity. The mathematical background needed to correctly interpret the output signals from nanomechanical biosensors is also outlined here. Other practical issues reviewed are the immobilization of bioreceptor molecules on the surface of nanomechanical sensors and methods to attain that in large arrays of sensors. Based on these phenomena, we describe different kinds of nanomechanical biosensors developed so far, providing some examples of the most relevant reported applications. Nanomechanical biosensors have demonstrated tremendous capabilities to target the recognition of biomolecular interactions through changes in surface stress or mass. Based on recent developments, this review points out that nanomechanical biosensors offer a unique asset to detect, in addition to the mass and surface stress, Young's modulus and viscoelasticity. The mathematical background needed to correctly interpret the output signals from nanomechanical biosensors and to attain deconvolution of these parameters is also outlined in this review. The simultaneous and sensitive tracking of diverse mechanical parameters opens fascinating opportunities to achieve a better understating of complex biological systems such as living cells, biomarkers in serum, biological machines, pathogen-drug interactions, and disease processes. Without any doubt, many challenging battlefronts must still be overcome that include: i) the development of nanomechanical systems that provide simultaneously static and dynamic information with high sensitivity and enable signal deconvolution, ii) harnessing the opportunities offered by measuring a high number of vibration modes, iii) to surmount the high loss of mechanical energy in liquids by novel driving strategies and/or specially designed nanomechanical systems, iv) full implementation of nanoscale resonators (including nanowires and nanotubes) in nanomechanical biosensor devices, v) the integration of the devices with microfluidics to purify and preconcentrate clinical samples, etc. This review aims at being a practical guide for successfully approaching this growing and fascinating field.

\section{Acknowledgements}

The authors would like to acknowledge financial support from the Spanish Science Ministry through projects ANEM! (TEC2009-14517-C02), INMUNO-swing (IPT-2011-0821-010000), POLYSENS (PIB2010BZ-0510) and ForceForFuture (CSD2010-00024); and from European Research Council through Starting Grant NANOFORCELLS (ERC-StG-2011-278860). 


\section{REFERENCES}

1 D'Orazio, P. Biosensors in clinical chemistry-2011 update. Clinica Chimica Acta (2011).

2 Tothill, I. E. 55-62 (Elsevier).

3 Justino, C. I. L., Rocha-Santos, T. A. \& Duarte, A. C. Review of analytical figures of merit of sensors and biosensors in clinical applications. TrAC Trends in Analytical Chemistry 29, 1172-1183 (2010).

4 Fan, X. et al. Sensitive optical biosensors for unlabeled targets: A review. analytica chimica acta 620, 8-26 (2008).

5 Wang, J. Carbon-nanotube based electrochemical biosensors: A review. Electroanalysis 17, 7-14 (2005).

6 Wang, J. Electrochemical biosensors: Towards point-of-care cancer diagnostics. Biosensors and Bioelectronics 21, 1887-1892 (2006).

7 Wang, J. Amperometric biosensors for clinical and therapeutic drug monitoring: a review. Journal of pharmaceutical and biomedical analysis 19, 47-53 (1999).

8 Stern, E. et al. Label-free biomarker detection from whole blood. Nature nanotechnology 5, 138-142 (2009).

9 Duan, X. et al. Quantification of the affinities and kinetics of protein interactions using silicon nanowire biosensors. Nature nanotechnology 7, 401-407 (2012).

10 Zheng, G., Gao, X. P. A. \& Lieber, C. M. Frequency domain detection of biomolecules using silicon nanowire biosensors. Nano Letters (2010).

11 Dixon, M. C. Quartz crystal microbalance with dissipation monitoring: enabling realtime characterization of biological materials and their interactions. Journal of biomolecular techniques: JBT 19, 151 (2008).

12 Länge, K., Rapp, B. E. \& Rapp, M. Surface acoustic wave biosensors: a review. Analytical and bioanalytical chemistry 391, 1509-1519 (2008).

13 O'sullivan, C. \& Guilbault, G. Commercial quartz crystal microbalances-theory and applications. Biosensors and Bioelectronics 14, 663-670 (1999).

14 Arlett, J., Myers, E. \& Roukes, M. Comparative advantages of mechanical biosensors. Nature nanotechnology 6, 203-215 (2011).

15 Boisen, A. \& Thundat, T. Design \& fabrication of cantilever array biosensors. Materials Today 12, 32-38 (2009).

16 Datar, R. et al. Cantilever sensors: nanomechanical tools for diagnostics. MRS bulletin 34, 449-454 (2009).

17 Boisen, A., Dohn, S., Keller, S. S., Schmid, S. \& Tenje, M. Cantilever-like micromechanical sensors. Reports on Progress in Physics 74, 036101 (2011).

18 Fritz, J. Cantilever biosensors. Analyst 133, 855-863 (2008).

19 Raiteri, R., Grattarola, M., Butt, H. J. \& Skládal, P. Micromechanical cantilever-based biosensors. Sensors and Actuators B: Chemical 79, 115-126 (2001).

20 Waggoner, P. S. \& Craighead, H. G. Micro-and nanomechanical sensors for environmental, chemical, and biological detection. Lab Chip 7, 1238-1255 (2007).

21 Boehr, D. D., Nussinov, R. \& Wright, P. E. The role of dynamic conformational ensembles in biomolecular recognition. Nature chemical biology 5, 789-796 (2009).

22 Savir, Y. \& Tlusty, T. Conformational proofreading: the impact of conformational changes on the specificity of molecular recognition. PLoS One 2, e468 (2007).

23 Browne, W. R. \& Feringa, B. L. Making molecular machines work. Nature nanotechnology 1, 25-35 (2006).

24 Itoh, H. et al. Mechanically driven ATP synthesis by F1-ATPase. Nature 427, 465-468 (2004).

25 Noji, H., Yasuda, R., Yoshida, M. \& Kinosita, K. Direct observation of the rotation of F1ATPase. Nature 386, 299-302 (1997). 
Hess, H. \& Bachand, G. D. Biomolecular motors. Materials Today 8, 22-29 (2005).

27 Zimmerberg, J. \& Kozlov, M. M. How proteins produce cellular membrane curvature. Nature Reviews Molecular Cell Biology 7, 9-19 (2005).

28 Baumgart, T., Capraro, B. R., Zhu, C. \& Das, S. L. Thermodynamics and mechanics of membrane curvature generation and sensing by proteins and lipids. Annual Review of Physical Chemistry 62, 483-506 (2011). cancer patients. Nature nanotechnology 2, 780-783 (2007).

Guck, J. et al. Optical deformability as an inherent cell marker for testing malignant transformation and metastatic competence. Biophysical journal 88, 3689-3698 (2005). Suresh, S. Biomechanics and biophysics of cancer cells. Acta Materialia 55, 3989-4014 (2007).

32 Michel, J. et al. Nanoindentation studies of full and empty viral capsids and the effects of capsid protein mutations on elasticity and strength. Proceedings of the National Academy of Sciences 103, 6184-6189 (2006).

33 Kol, N. et al. A stiffness switch in human immunodeficiency virus. Biophysical journal 92, 1777-1783 (2007).

34 Hansen, K. M. \& Thundat, T. Microcantilever biosensors. Methods 37, 57-64 (2005).

35 Goeders, K. M., Colton, J. S. \& Bottomley, L. A. Microcantilevers: sensing chemical interactions via mechanical motion. Chemical reviews 108, 522 (2008). Yoshikawa, G., Akiyama, T., Gautsch, S., Vettiger, P. \& Rohrer, H. Nanomechanical Membrane-type Surface Stress Sensor. Nano Letters (2011).

37 Waggoner, P. S., Varshney, M. \& Craighead, H. G. Detection of prostate specific antigen with nanomechanical resonators. Lab Chip 9, 3095-3099 (2009).

38 Schmid, S., Jensen, K., Nielsen, K. \& Boisen, A. Damping mechanisms in high-Q micro and nanomechanical string resonators. Physical Review B 84, 165307 (2011).

39 Tamayo, J. et al. Imaging the surface stress and vibration modes of a microcantilever by laser beam deflection microscopy. Nanotechnology 23, 315501 (2012). Favero, I. \& Karrai, K. Optomechanics of deformable optical cavities. Nature Photonics 3, 201-205 (2009).

41 Kippenberg, T. J. \& Vahala, K. J. Cavity optomechanics: Back-action at the mesoscale. Science 321, 1172-1176 (2008).

42 Ramos, D. et al. Optomechanics with Silicon Nanowires by Harnessing Confined Electromagnetic Modes. Nano Letters (2012).

43 Godin, M. et al. Cantilever-based sensing: the origin of surface stress and optimization strategies. Nanotechnology 21, 075501 (2010).

44 Ibach, H. Adsorbate-induced surface stress. Journal of Vacuum Science \& Technology A: Vacuum, Surfaces, and Films 12, 2240-2245 (1994).

45 Haiss, W. Surface stress of clean and adsorbate-covered solids. Reports on Progress in Physics 64, 591 (2001).

46 Zhang, J. et al. Rapid and label-free nanomechanical detection of biomarker transcripts in human RNA. Nature nanotechnology 1, 214-220 (2006).

47 McKendry, R. et al. Multiple label-free biodetection and quantitative DNA-binding assays on a nanomechanical cantilever array. Proceedings of the National Academy of Sciences 99, 9783 (2002).

48 Mader, A. et al. Discrimination of Escherichia coli Strains using Glycan Cantilever Array Sensors. Nano Letters (2012).

49 Mertens, J. et al. Label-free detection of DNA hybridization based on hydrationinduced tension in nucleic acid films. Nature nanotechnology 3, 301-307 (2008).

50 Gruber, K. et al. Cantilever Array Sensors Detect Specific Carbohydrate-Protein Interactions with Picomolar Sensitivity. ACS nano (2011). 
51 Arntz, Y. et al. Label-free protein assay based on a nanomechanical cantilever array. Nanotechnology 14, 86 (2003).

52 Alvarez, M. et al. Development of nanomechanical biosensors for detection of the pesticide DDT. Biosensors and Bioelectronics 18, 649-653 (2003).

53 Mertens, J., Daudén, M. I., Carrascosa, J. L. \& Tamayo, J. Interaction of viral ATPases with nucleotides measured with a microcantilever. Sensors and Actuators B: Chemical (2012).

54 Shu, W. et al. DNA molecular motor driven micromechanical cantilever arrays. Journal of the American Chemical Society 127, 17054-17060 (2005).

55 Sader, J. E. Surface stress induced deflections of cantilever plates with applications to the atomic force microscope: Rectangular plates. Journal of applied physics $89,2911-$ 2921 (2001).

56 Stoney, G. G. The tension of metallic films deposited by electrolysis. Proceedings of the Royal Society of London. Series A, Containing Papers of a Mathematical and Physical Character 82, 172-175 (1909).

57 Weissmüller, J. \& Duan, H. Cantilever bending with rough surfaces. Physical review letters 101, 146102 (2008).

58 Mohammadi, P. \& Sharma, P. Atomistic elucidation of the effect of surface roughness on curvature-dependent surface energy, surface stress, and elasticity. Applied physics letters 100, 133110-133110-133114 (2012).

59 Ergincan, O., Palasantzas, G. \& Kooi, B. Influence of random roughness on cantilever curvature sensitivity. Applied physics letters 96, 041912 (2010).

60 Lachut, M. J. \& Sader, J. E. Effect of surface stress on the stiffness of thin elastic plates and beams. Physical Review B 85, 085440 (2012).

61 Karabalin, R., Villanueva, L., Matheny, M., Sader, J. \& Roukes, M. Stress-Induced Variations in the Stiffness of Micro-and Nanocantilever Beams. Physical review letters 108, 236101 (2012).

62 Lagowski, J., Gatos, H. \& Sproles, E. Surface stress and the normal mode of vibration of thin crystals: GaAs. Applied physics letters 26, 493-495 (1975).

63 Gurtin, M., Markenscoff, X. \& Thurston, R. Effect of surface stress on the natural frequency of thin crystals. Applied physics letters 29, 529 (1976).

64 Lu, P., Lee, H., Lu, C. \& O'shea, S. Surface stress effects on the resonance properties of cantilever sensors. Physical Review B 72, 085405 (2005).

65 Chen, G., Thundat, T., Wachter, E. \& Warmack, R. Adsorption-induced surface stress and its effects on resonance frequency of microcantilevers. Journal of applied physics 77, 3618-3622 (1995).

66 Lachut, M. J. \& Sader, J. E. Effect of surface stress on the stiffness of cantilever plates. Physical review letters 99, 206102 (2007).

67 Pini, V. et al. Shedding Light on Axial Stress Effect on Resonance Frequencies of Nanocantilevers. ACS nano (2011).

68 Venstra, W. J., Westra, H. J. R. \& van der Zant, H. S. J. Mechanical stiffening, bistability, and bit operations in a microcantilever. Applied physics letters 97, 193107 (2010).

69 V. Pini, J. J. R., M. Calleja and J. Tamayo. Effect of surface stress on the eigenfrequencies of resonant beams. (2012).

70 Ilic, B. et al. Attogram detection using nanoelectromechanical oscillators. Journal of applied physics 95, 3694-3703 (2004).

71 Ekinci, K., Huang, X. \& Roukes, M. Ultrasensitive nanoelectromechanical mass detection. Applied physics letters 84, 4469-4471 (2004).

72 Lassagne, B., Garcia-Sanchez, D., Aguasca, A. \& Bachtold, A. Ultrasensitive mass sensing with a nanotube electromechanical resonator. Nano Letters 8, 3735-3738 (2008). 

Nature nanotechnology 3, 533-537 (2008).

74 Chiu, H. Y., Hung, P., Postma, H. W. C. \& Bockrath, M. Atomic-scale mass sensing using carbon nanotube resonators. Nano Letters 8, 4342-4346 (2008).

75 Chaste, J. et al. A nanomechanical mass sensor with yoctogram resolution. Nature nanotechnology 7, 301-304 (2012).

76 Van Eysden, C. A. \& Sader, J. E. Frequency response of cantilever beams immersed in viscous fluids with applications to the atomic force microscope: Arbitrary mode order. Journal of applied physics 101, 044908-044908-044911 (2007).

77 Sader, J. E. Frequency response of cantilever beams immersed in viscous fluids with applications to the atomic force microscope. Journal of applied physics 84, 64 (1998). Grüter, R. et al. Disentangling mechanical and mass effects on nanomechanical resonators. Applied physics letters 96, 023113 (2010).

79 Sushko, M. L., Harding, J. H., Shluger, A. L., McKendry, R. A. \& Watari, M. Physics of nanomechanical biosensing on cantilever arrays. Advanced Materials 20, 3848-3853 (2008).

80 Ramos, D., Tamayo, J., Mertens, J., Calleja, M. \& Zaballos, A. Origin of the response of nanomechanical resonators to bacteria adsorption. Journal of applied physics 100, 106105 (2006).

81 Ramos, D. et al. Detection of bacteria based on the thermomechanical noise of a nanomechanical resonator: origin of the response and detection limits. Nanotechnology 19, 035503 (2008).

82 Craighead, H. Nanomechanical systems: Measuring more than mass. Nature nanotechnology 2, 18-19 (2007).

83 Tamayo, J., Ramos, D., Mertens, J. \& Calleja, M. Effect of the adsorbate stiffness on the resonance response of microcantilever sensors. Applied physics letters 89, 224104 (2006).

84 Zhao, Y., Ge, Z. \& Fang, J. Elastic modulus of viral nanotubes. Physical Review E 78, 031914 (2008).

85 Schmatulla, A., Maghelli, N. \& Marti, O. Micromechanical properties of tobacco mosaic viruses. Journal of Microscopy 225, 264-268 (2007).

86 Calleja, M., Tamayo, J., Nordstrom, M. \& Boisen, A. Low-noise polymeric nanomechanical biosensors. Applied Physics Letters 88, 113901-113901-113903 (2006).

87 Höök, F. et al. Variations in coupled water, viscoelastic properties, and film thickness of a Mefp-1 protein film during adsorption and cross-linking: a quartz crystal microbalance with dissipation monitoring, ellipsometry, and surface plasmon resonance study. Analytical chemistry 73, 5796-5804 (2001).

88 Höök, F., Rodahl, M., Brzezinski, P. \& Kasemo, B. Energy dissipation kinetics for protein and antibody-antigen adsorption under shear oscillation on a quartz crystal microbalance. Langmuir 14, 729-734 (1998).

89 Jung, N. \& Jeon, S. Nanomechanical thermal analysis with silicon cantilevers of the mechanical properties of poly (vinyl acetate) near the glass transition temperature. Macromolecules 41, 9819-9822 (2008).

90 Dufour, I., Lochon, F., Heinrich, S. M., Josse, F. \& Rebiere, D. Effect of coating viscoelasticity on quality factor and limit of detection of microcantilever chemical sensors. Sensors Journal, IEEE 7, 230-236 (2007).

91 Sawano, S., Arie, T. \& Akita, S. Carbon nanotube resonator in liquid. Nano Letters (2010).

92 Verbridge, S. S., Bellan, L. M., Parpia, J. M. \& Craighead, H. Optically driven resonance of nanoscale flexural oscillators in liquid. Nano Letters 6, 2109-2114 (2006). 

sensing in dissipative media. Journal of Micromechanics and Microengineering 21, 065002 (2011).

94 Cagliani, A., Kosaka, P., Tamayo, J. \& Davis, Z. J. Monitoring the hydration of DNA selfassembled monolayers using an extensional nanomechanical resonator. Lab on a Chip (2012).

95 Cagliani, A. \& Davis, Z. Ultrasensitive bulk disk microresonator-based sensor for distributed mass sensing. Journal of Micromechanics and Microengineering 21, 045016 (2011).

96 Nicu, L. \& Leichle, T. Biosensors and tools for surface functionalization from the macroto the nanoscale: The way forward. Journal of applied physics 104, 111101 (2008).

97 Vericat, C., Vela, M., Benitez, G., Carro, P. \& Salvarezza, R. Self-assembled monolayers of thiols and dithiols on gold: new challenges for a well-known system. Chem. Soc. Rev. 39, 1805-1834 (2010).

98 Berger, R. et al. Surface stress in the self-assembly of alkanethiols on gold. Science 276, 2021 (1997).

99 Fritz, J. et al. Translating biomolecular recognition into nanomechanics. Science 288, 316-318 (2000).

100 Álvarez, M. et al. Nanomechanics of the formation of DNA self-assembled monolayers and hybridization on microcantilevers. Langmuir 20, 9663-9668 (2004).

101 Ramos, D. et al. Arrays of dual nanomechanical resonators for selective biological detection. Analytical chemistry 81, 2274-2279 (2009).

$102 \mathrm{Wu}$, G. et al. Bioassay of prostate-specific antigen (PSA) using microcantilevers. Nature biotechnology 19, 856-860 (2001).

103 Rye, R., Nelson, G. \& Dugger, M. Mechanistic aspects of alkylchlorosilane coupling reactions. Langmuir 13, 2965-2972 (1997).

104 Pesek, J. J. \& Matyska, M. T. Methods for the modification and characterization of oxide surfaces. Interface Science 5, 103-117 (1997).

105 Varshney, M., Waggoner, P. S., Montagna, R. A. \& Craighead, H. G. Prion protein detection in serum using micromechanical resonator arrays. Talanta 80, 593-599 (2009).

106 Martínez, N. et al. High throughput optical readout of dense arrays of nanomechanical systems for sensing applications. Review of Scientific Instruments 81, 125109-125109125109 (2010).

107 Braun, T. et al. Quantitative time-resolved measurement of membrane protein-ligand interactions using microcantilever array sensors. Nature nanotechnology 4, 179-185 (2009).

108 Bietsch, A., Zhang, J., Hegner, M., Lang, H. P. \& Gerber, C. Rapid functionalization of cantilever array sensors by inkjet printing. Nanotechnology 15, 873 (2004).

109 Lukacs, G., Maloney, N. \& Hegner, M. Ink-jet printing: perfect tool for cantilever array sensor preparation for microbial growth detection. Journal of Sensors 2012 (2011).

110 Guillon, S. et al. Biological functionalization of massively parallel arrays of nanocantilevers using microcontact printing. Sensors and Actuators B: Chemical (2011).

111 Ndieyira, J. W. et al. Nanomechanical detection of antibiotic-mucopeptide binding in a model for superbug drug resistance. Nature nanotechnology 3, 691-696 (2008).

112 Watari, M. et al. Investigating the molecular mechanisms of in-plane mechanochemistry on cantilever arrays. Journal of the American Chemical Society 129, 601-609 (2007).

113 Hagan, M. F., Majumdar, A. \& Chakraborty, A. K. Nanomechanical forces generated by surface grafted DNA. The Journal of Physical Chemistry B 106, 10163-10173 (2002). 
114 Sanii, B. \& Ashby, P. D. High sensitivity deflection detection of nanowires. Physical review letters 104, 147203 (2010).

115 Ilic, B., Yang, Y. \& Craighead, H. Virus detection using nanoelectromechanical devices. Applied physics letters 85, 2604 (2004).

116 Ilic, B. et al. Enumeration of DNA molecules bound to a nanomechanical oscillator. Nano Letters 5, 925-929 (2005).

117 Naik, A., Hanay, M., Hiebert, W., Feng, X. \& Roukes, M. Towards single-molecule nanomechanical mass spectrometry. Nature nanotechnology 4, 445-450 (2009).

118 Ilic, B. et al. Mechanical resonant immunospecific biological detector. Applied physics letters 77, 450 (2000).

119 Ilic, B. et al. Single cell detection with micromechanical oscillators. Journal of Vacuum Science \& Technology B: Microelectronics and Nanometer Structures 19, 2825 (2001).

120 Johnson, L., Gupta, A. K., Ghafoor, A., Akin, D. \& Bashir, R. Characterization of vaccinia virus particles using microscale silicon cantilever resonators and atomic force microscopy. Sensors and Actuators B: Chemical 115, 189-197 (2006).

121 Braun, T. et al. Micromechanical mass sensors for biomolecular detection in a physiological environment. Physical Review E 72, 031907 (2005).

122 Ramos, D., Mertens, J., Calleja, M. \& Tamayo, J. Phototermal self-excitation of nanomechanical resonators in liquids. Applied physics letters 92, 173108 (2008).

123 Ghatkesar, M. K. et al. Resonating modes of vibrating microcantilevers in liquid. Applied physics letters 92, 043106 (2008).

124 Tamayo, J., Calleja, M., Ramos, D. \& Mertens, J. Underlying mechanisms of the selfsustained oscillation of a nanomechanical stochastic resonator in a liquid. Physical Review B 76, 180201 (2007).

125 Bantscheff, M., Schirle, M., Sweetman, G., Rick, J. \& Kuster, B. Quantitative mass spectrometry in proteomics: a critical review. Analytical and bioanalytical chemistry 389, 1017-1031 (2007).

126 Benesch, J. L. P. \& Robinson, C. V. Mass spectrometry of macromolecular assemblies: preservation and dissociation. Current opinion in structural biology 16, 245-251 (2006).

127 Gil-Santos, E. et al. Nanomechanical mass sensing and stiffness spectrometry based on two-dimensional vibrations of resonant nanowires. Nature nanotechnology 5, 641-645 (2010).

128 Burg, T. P. et al. Vacuum-packaged suspended microchannel resonant mass sensor for biomolecular detection. Microelectromechanical Systems, Journal of 15, 1466-1476 (2006).

129 Burg, T. P. et al. Weighing of biomolecules, single cells and single nanoparticles in fluid. Nature 446, 1066-1069 (2007).

130 Grover, W. H. et al. Measuring single-cell density. Proceedings of the National Academy of Sciences 108, 10992 (2011).

131 Oesterschulze, E. et al. Tailoring the interface of hybrid microresonators in viscid fluids enhances their quality factor by two orders of magnitude. Lab Chip (2012).

132 Melli, M., Scoles, G. \& Lazzarino, M. Fast Detection of Biomolecules in Diffusion Limited Regime Using Micromechanical Pillars. ACS nano (2011).

133 Sadeghian, H. et al. 631-634 (IEEE).

134 Sadeghian, H., Goosen, H., Bossche, A. \& Van Keulen, F. Application of electrostatic pull-in instability on sensing adsorbate stiffness in nanomechanical resonators. Thin Solid Films 518, 5018-5021 (2010).

135 Cleland, A. \& Roukes, M. Noise processes in nanomechanical resonators. Journal of applied physics 92, 2758-2769 (2002).

136 Alvarez, M. et al. Dimension dependence of the thermomechanical noise of microcantilevers. Journal of applied physics 99, 024910 (2006). 
137 Ekinci, K., Yang, Y. \& Roukes, M. Ultimate limits to inertial mass sensing based upon nanoelectromechanical systems. Journal of applied physics 95, 2682 (2004).

138 Rast, S., Wattinger, C., Gysin, U. \& Meyer, E. The noise of cantilevers. Nanotechnology 11, 169 (2000).

139 Albrecht, T., Grutter, P., Horne, D. \& Rugar, D. Frequency modulation detection using high-Q cantilevers for enhanced force microscope sensitivity. Journal of applied physics 69, 668-673 (1991).

140 Nair, P. R. \& Alam, M. A. Theory of "Selectivity" of label-free nanobiosensors: A geometro-physical perspective. Journal of applied physics 107, 064701-064701-064706 (2010).

141 Priscila M. Kosaka, J. T., José J. Ruz, Sara Puertas, Ester Polo, Valeria Grazu, Jesús M. de la Fuente and Montserrat Calleja. A Statistical Approach to achieve highly Sensitive and Specific End-point detection of Immunoreactions by Nanomechanical Biosensors (2012). 